\title{
Influence of initial stress, irregularity and heterogeneity on Love-type wave propagation in double pre-stressed irregular layers lying over a pre-stressed half-space
}

\author{
Abhishek Kumar Singh, Amrita Das*, Zeenat Parween and Amares Chattopadhyay \\ Department of Applied Mathematics, Indian School of Mines, Dhanbad 826 004, Jharkhand, India. \\ ${ }^{*}$ Corresponding author.e-mail: amritadas.ism@gmail.com
}

The present paper deals with the propagation of Love-type wave in an initially stressed irregular vertically heterogeneous layer lying over an initially stressed isotropic layer and an initially stressed isotropic halfspace. Two different types of irregularities, viz., rectangular and parabolic, are considered at the interface of uppermost initially stressed heterogeneous layer and intermediate initially stressed isotropic layer. Dispersion equations are obtained in closed form for both cases of irregularities, distinctly. The effect of size and shape of irregularity, horizontal compressive initial stress, horizontal tensile initial stress, heterogeneity of the uppermost layer and width ratio of the layers on phase velocity of Love-type wave are the major highlights of the study. Comparative study has been made to identify the effects of different shapes of irregularity, presence of heterogeneity and initial stresses. Numerical computations have been carried out and depicted by means of graphs for the present study.

\section{Introduction}

Regulation of seismic wave propagation due to structural deformations of the boundary of elastic medium, in which it is propagating, is an undeniable reality. Consequently, seismologists and geophysicists found it significant to study the propagation of seismic waves in an elastic medium with divergent irregularities in order to understand and predict the seismic behaviour at different mountain basins of the earth, continental margins, etc. Chattopadhyay et al. (2011a, b) proposed the idea of finding the dispersion equation of magnetoelastic shear waves in an irregular monoclinic layer. Chattopadhyay et al. (1983) studied the propagation of an SH-guided wave in an internal stratum with a parabolic irregularity in the lower interface. Chattopadhyay and De (1983) studied the propagation of Love waves in a porous layer underlain by isotropic elastic medium with a rectangular irregularity at the interface. Chattopadhyay and Pal (1983) established the dispersion relation of $\mathrm{SH}$-waves caused by irregularity in the pre-stressed internal stratum. Acharya and Roy (2009) investigated the plane $\mathrm{SH}$ waves through a magnetoelastic crustal layer based over an elastic, solid semi-space under the influence of surface stress on the free surface of the crustal layer and irregularity of the interface. Singh (2011) discussed the propagation of Love wave in a layer medium bounded by irregular boundary surfaces as a function of the amplitudes of the corrugation, frequency and position parameters of the corrugated boundary surfaces. Ben-Hador and Buchen (1999) have established the dispersion of Love and Rayleigh waves in multi-layered models with smooth and weakly nonparallel boundaries using the first-order perturbation theory of Whitham's equation. Propagation of a Love wave in an initially stressed fluid-saturated anisotropic porous layer with an irregular boundary

Keywords. Initial stress; irregularity; non-homogeneous; Love-type wave; isotropic; double layer. 
sandwiched between two isotropic half-spaces was studied by Chattaraj et al. (2012). Chattopadhyay et al. (2011a, b) studied the propagation of horizontally polarized shear waves in an internal magnetoelastic monoclinic stratum with irregularity in lower interface. Chattopadhyay and Singh (2012) studied the effect of irregularity on the propagation of horizontally polarized shear waves in an irregular magneto-elastic self-reinforced stratum sandwiched between two semi-infinite magneto-elastic self-reinforced media considering two shapes of irregularities namely rectangular and parabolic on the interface of layer and lower semi-infinite media.

The factors such as overburdened layer, variation in temperature, slow process of creep and gravitational field, etc., have pronounced influence on the propagation of waves as they are responsible for the evolution of a large proportion of initial stress in a medium. Comprehensive information can be earned from the disquisition of Biot (1965). A medium which can remold the attitude of the medium due to some physical or mechanical obligations is designated as pre-stressed medium whose occupancy may increase or decrease the overall rigidity of an elastic structure. A state of initial stress (pre-stress) in a deformable medium induces mechanical properties which depend mainly on the magnitude of the stress and are quite distinct from those associated with the rigidity of the material itself. Our earth is a highly initially stressed medium. Dey and Addy (1978) have shown the effect of initial stresses on the propagation of Love waves by considering the layer and the half-space to be isotropic elastic in one case and visco-elastic in another case. Acharya et al. (2009) employed elasto-dynamical equations for transversely isotropic solids to investigate the general theory of transversely isotropic magneto-elastic interface waves in conducting media under initial hydrostatic tension or compression. Recently, the dynamical response of normal moving load on an irregular fiber-reinforced half-space is discussed by Kaur et al. (2014). Khurana and Vashisth (2001) derived the frequency of Love waves propagating in a pre-stressed elastic layer overlying a poro-elastic solid half-space having a loosely bounded common interface.

Natural media (atmosphere, ocean, ground and biological medium) exhibit widespread and often time varying non-homogeneities due to the spatial dependence in their material properties. These non-homogeneities may be further accompanied by layering anisotropy and the presence of inclusions. Accordingly, it became an attractive proposition to represent a particular medium as a homogeneous matrix over which certain properties such as density, bulk modulus, etc., exhibit a random variation. The sort of non-homogeneity existing in the earth affects the propagation of seismic waves in such media. Therefore, the critique of propagation of seismic waves in heterogeneous medium has gained much gravity within the province of applied mathematics and engineering. Some valuable information about the propagation of seismic waves is available in Ewing and Press (1957). Numerous leading researchers gave thoughtful upshots on the theory of Love-type wave propagation in a medium where the velocity, rigidity and density are functions of depth. Dutta (1963) discussed a problem relating to the propagation of Love-type waves in a heterogeneous internal stratum lying between two semi-infinite homogeneous elastic media. Bhattacharya (1969) discussed the possibility of the propagation of Love waves in an intermediate heterogeneous layer where the heterogeneity in rigidity was assumed as exponentially varying function of depth in one case and linearly varying function of depth in another case. Sato (1952) studied the propagation of $\mathrm{SH}$ waves in a double superficial layer over heterogeneous medium by taking variation in rigidity. Till date no attempt has been made to study the propagation of Lovetype wave in double layers of finite width containing an irregularity at the common interface and lying over a half-space. Inclusion of initial stress in each medium, heterogeneity and different types of irregularity make the present study more relevant towards its practical implications.

Since our earth is a layered structure, and heterogeneity and initial stress are its trivial characteristics, the real scenario beneath the surface of earth itself is a good model for considering double prestressed layer with an irregular interface lying over a pre-stressed half-space. Further, initial stresses occur in structural elements during their manufacture and assembly, in the earth's crust under the action of geostatic and geodynamic forces, in composites when they are created, in rock, etc. Moreover, the evidence for the fact that 'elastic constants of a layer may be functions of depth' is also available. Apart from these, presence of irregular boundary surfaces of the constituent layers of earth due to the presence of salt and ore deposits deep beneath the earth, mountains, basins, mountain roots, etc., is unavoidable. All these factors greatly affect the propagation of seismic wave through earth.

The present study concerns the propagation of Love-type wave in an initially stressed irregular vertically heterogeneous layer overlying an initially stressed isotropic layer and an initially stressed isotropic half-space. Heterogeneity in the uppermost layer is caused due to initial stress, rigidity and density which are exponentially varying functions of depth. Dispersion relations are found in closed form for the case of rectangular irregularity 
and parabolic irregularity separately. The effect of irregularity parameter, horizontal compressive initial stress, horizontal tensile initial stress, heterogeneity parameters, width ratio of the layers on the phase velocity are the major outcomes of the study and these are depicted graphically. Comparative study has also been made to trace out some of the important peculiarities of the study.

\section{Formulation of the problem}

We consider the propagation of Love-type wave in an initially stressed heterogeneous irregular isotropic layer $\left(M_{1}\right)$ lying over an initially stressed isotropic layer $\left(M_{2}\right)$ and an initially stressed isotropic half-space $\left(M_{3}\right)$. Cartesian co-ordinate system has been chosen in such a way that $z$-axis is pointing positively downwards and $x$-axis is in the direction of wave propagation, lying along the common interface of medium $M_{2}$ and $M_{3}$. Two different types of irregularities have been assumed, viz., rectangular and parabolic as shown in figures 1 and 2 respectively, with span $2 s$ and depth $H^{\prime}$, at the interface of uppermost initially stressed heterogeneous layer and intermediate initially stressed isotropic layer. $H_{1}$ is the thickness of the intermediate layer whereas $\left(H_{2}-H_{1}\right)$ is the thickness of the uppermost layer. $O$ is the origin of the rectangular co-ordinate system. The source of disturbance $S$, is placed on the positive $z$-axis at a distance $d\left(>H^{\prime}\right)$ from the origin. The equation of the interface may be defined as:

$$
z=\varepsilon h(x)-H_{1}
$$

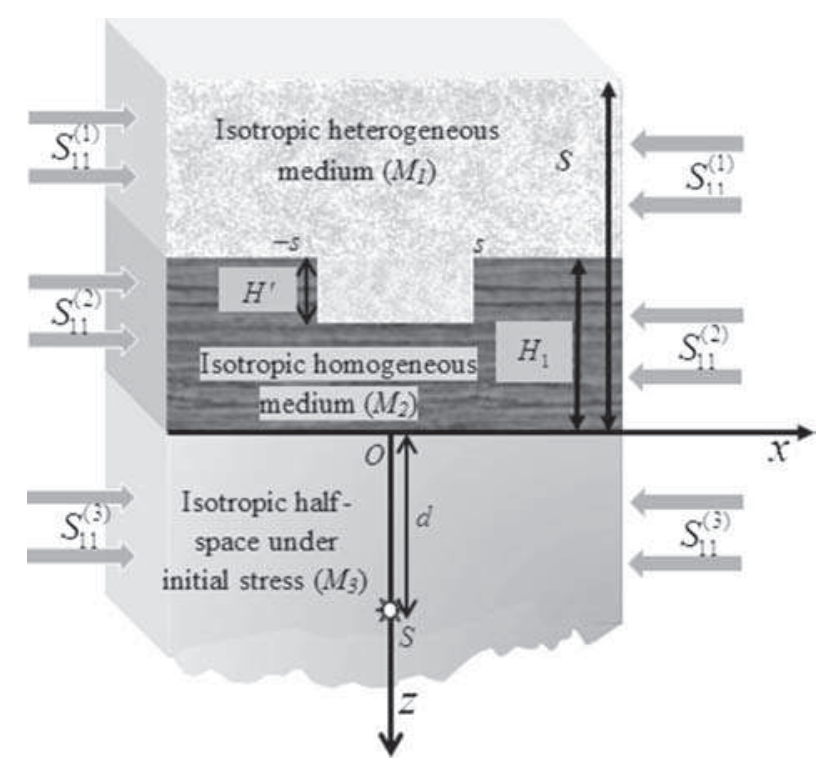

Figure 1. Geometry of the problem with rectangular irregularity. where

$$
h(x)= \begin{cases}2 s, & |x|<s \\ 0, & |x| \geq s\end{cases}
$$

for the case of rectangular irregularity as shown in figure 1 and

$$
h(x)= \begin{cases}2 s\left(1-\frac{x^{2}}{s^{2}}\right), & |x|<s \\ 0, & |x| \geq s\end{cases}
$$

for the case of parabolic irregularity as shown in figure 2.

Here, $\varepsilon=\left(H^{\prime} / 2 s\right) \ll 1$, known as perturbation parameter, is a small positive number under the assumption that span of the irregularity is very large compared to the depth of the irregularity.

For the propagation of Love-type wave, we consider:

$$
u_{i}=0, \quad w_{i}=0, \quad v_{i}=v_{i}(x, z, t), \quad i=1,2,3 .
$$

The heterogeneity in the uppermost layer is taken as:

$$
\mu_{1}=\mu_{1}^{0} e^{v z}, \quad \rho_{1}=\rho_{1}^{0} e^{v z}, \quad S_{11}^{(1)}=S_{11}^{(0)} e^{v z},
$$

where $v$ is the heterogeneity parameter. In the absence of body force, the equation of motion for the uppermost heterogeneous layer with initial stress $S_{11}^{(1)}$ is obtained as:

$$
\left(1+\xi_{1}\right) \frac{\partial^{2} v_{1}}{\partial x^{2}}+\frac{\partial^{2} v_{1}}{\partial z^{2}}+v \frac{\partial v_{1}}{\partial z}=\frac{1}{\beta_{1}^{2}} \frac{\partial^{2} v_{1}}{\partial t^{2}}
$$

where

$$
\beta_{1}=\sqrt{\frac{\mu_{1}^{0}}{\rho_{1}^{0}}} \quad \text { and } \quad \xi_{1}=\frac{S_{11}^{(0)}}{2 \mu_{1}^{0}} .
$$

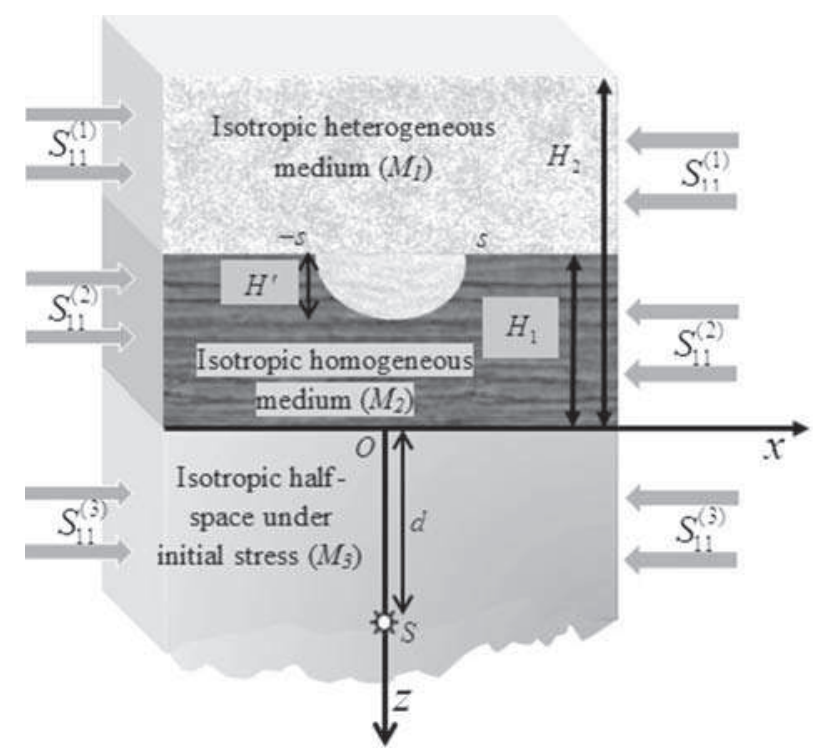

Figure 2. Geometry of the problem with parabolic irregularity. 
In the absence of body force, the equation of motion for the intermediate isotropic layer with initial stress $S_{11}^{(2)}$ is obtained as:

$$
\left(1+\xi_{2}\right) \frac{\partial^{2} v_{2}}{\partial x^{2}}+\frac{\partial^{2} v_{2}}{\partial z^{2}}=\frac{1}{\beta_{2}^{2}} \frac{\partial^{2} v_{2}}{\partial t^{2}},
$$

where

$$
\beta_{2}=\sqrt{\frac{\mu_{2}}{\rho_{2}}} \quad \text { and } \quad \xi_{2}=\frac{S_{11}^{(2)}}{2 \mu_{2}} .
$$

The equation of motion, in the absence of body force, for the lowermost isotropic half-space is calculated as:

$$
\left(1+\xi_{3}\right) \frac{\partial^{2} v_{3}}{\partial x^{2}}+\frac{\partial^{2} v_{3}}{\partial z^{2}}=\frac{1}{\beta_{3}^{2}} \frac{\partial^{2} v_{3}}{\partial t^{2}},
$$

where

$$
\beta_{3}=\sqrt{\frac{\mu_{3}}{\rho_{3}}} \quad \text { and } \quad \xi_{3}=\frac{S_{11}^{(3)}}{2 \mu_{3}} .
$$

The boundary conditions are as follows:

(i) Upper surface of the uppermost heterogeneous layer is stress free:

$$
\mu_{1}^{0} \frac{\partial v_{1}}{\partial z} e^{v z}=0 \quad \text { at } z=-H_{2} .
$$

(ii) Displacements are continuous at the interface:

$$
v_{1}=v_{2} \quad \text { at } z=\varepsilon h(x)-H_{1} .
$$

(iii) Stresses are continuous at the interface:

$$
\begin{gathered}
\mu_{1}^{0}\left(\frac{\partial v_{1}}{\partial z}-\varepsilon h^{\prime} \frac{\partial v_{1}}{\partial x}\right) e^{v z}=\mu_{2}\left(\frac{\partial v_{2}}{\partial z}-\varepsilon h^{\prime} \frac{\partial v_{2}}{\partial x}\right) \\
\text { at } z=\varepsilon h(x)-H_{1}, \text { where } \\
h^{\prime}=\frac{d h}{d x} .
\end{gathered}
$$

(iv) Displacements are continuous at the interface:

$$
v_{2}=v_{3} \quad \text { at } z=0 .
$$

(v) Stresses are continuous at the interface:

$$
\mu_{2} \frac{\partial v_{2}}{\partial z}=\mu_{3} \frac{\partial v_{3}}{\partial z} \quad \text { at } z=0 .
$$

\section{Solution of the problem}

Let us take the solution of equations (5), (6) and (7) as:

$$
v_{\zeta}(x, z, t)=V_{\zeta}(z, x) e^{i \omega t}, \quad(\zeta=1,2,3)
$$

where $\omega$ is the circular frequency.
Now, defining the Fourier transform $\overline{V_{\zeta}}(z, \eta)$ of $V_{\zeta}(z, x)$ as:

$$
\overline{V_{\zeta}}(z, \eta)=\int_{-\infty}^{\infty} V_{\zeta}(z, x) e^{i \eta x} d x
$$

and the inverse Fourier transform as:

$$
V_{\zeta}(z, x)=\frac{1}{2 \pi} \int_{-\infty}^{\infty} \overline{V_{\zeta}}(z, \eta) e^{-i \eta x} d \eta
$$

and taking the Fourier transform of equations (5), (6) and (7), we get:

$$
\frac{d^{2} \overline{V_{1}}}{d z^{2}}+v \frac{d \overline{V_{1}}}{d z}+p_{1}^{2} \overline{V_{1}}=0,
$$

$\frac{d^{2} \overline{V_{2}}}{d z^{2}}+p_{2}^{2} \overline{V_{2}}=0$

$$
\frac{d^{2} \overline{V_{3}}}{d z^{2}}-p_{3}^{2} \overline{V_{3}}=0
$$

where

$$
\begin{aligned}
& p_{1}^{2}=\left(\frac{\omega^{2}}{\beta_{1}^{2}}-\eta^{2}\left(1-\xi_{1}\right)\right), \\
& p_{2}^{2}=\left(\frac{\omega^{2}}{\beta_{2}^{2}}-\eta^{2}\left(1-\xi_{2}\right)\right), \\
& p_{3}^{2}=\left(\eta^{2}\left(1-\xi_{3}\right)-\frac{\omega^{2}}{\beta_{3}^{2}}\right) .
\end{aligned}
$$

The appropriate solutions of equations (11), (12) and (13) are

$$
\overline{V_{1}}=e^{-\frac{v}{2} z}(A \cos T z+B \sin T z),
$$

$$
\overline{V_{2}}=C \cos p_{2} z+D \sin p_{2} z,
$$

$$
\overline{V_{3}}=E e^{-p_{3} z},
$$

where

$$
T=\frac{1}{2} \sqrt{-v^{2}+4\left(\eta^{2}(1+\xi)-\frac{\omega^{2}}{\beta_{1}^{2}}\right)} .
$$

Therefore, the displacements in the three media are:

$V_{1}(z, x)=\frac{1}{2 \pi} \int_{-\infty}^{\infty}(A \cos T z+B \sin T z) e^{-\frac{v}{2} z} e^{-i \eta x} d \eta$, 
$V_{2}(z, x)=\frac{1}{2 \pi} \int_{-\infty}^{\infty}\left(C \cos p_{2} z+D \sin p_{2} z\right) e^{-i \eta x} d \eta$

$V_{3}(z, x)=\frac{1}{2 \pi} \int_{-\infty}^{\infty}\left(E e^{-p_{3} z}+\frac{2}{p_{3}} e^{p_{3} z} e^{-p_{3} d}\right) e^{-i \eta x} d \eta$

where the second term in the integrand of $V_{3}$ is introduced due to the source in the lowermost medium.

Since the interface of the uppermost and intermediate layer is not uniform, the terms $A, B, C$ and $D$ are also functions of the perturbation parameter, $\varepsilon$. Expanding these terms in ascending powers of $\varepsilon$ and retaining the terms up to the first order of $\varepsilon$, as $\varepsilon$ is a small parameter; the following approximations can be used:

$$
\begin{array}{ll}
A \cong A_{0}+A_{1} \varepsilon, & B \cong B_{0}+B_{1} \varepsilon, \\
C \cong C_{0}+C_{1} \varepsilon, & D \cong D_{0}+D_{1} \varepsilon, \\
e^{ \pm v \varepsilon h} \cong 1 \pm v \varepsilon h, & \cos v \varepsilon h \cong 1, \\
\sin v \varepsilon h \cong v \varepsilon h, &
\end{array}
$$

where $v$ is any quantity.

Using the boundary condition (i), we get:

$$
\begin{aligned}
& \left(A_{0} T \sin T H_{2}+B_{0} T \cos T H_{2}-\frac{v}{2} A_{0} \cos T H_{2}\right. \\
& \left.+\frac{v}{2} B_{0} \sin T H_{2}\right)+\varepsilon\left(A_{1} T \sin T H_{2}+B_{1} T \cos T H_{2}\right. \\
& \left.\quad-\frac{v}{2} A_{1} \cos T H_{2}+\frac{v}{2} B_{1} \sin T H_{2}\right)=0
\end{aligned}
$$

Using the boundary condition (ii), we have:

$$
\begin{aligned}
\int_{-\infty}^{\infty}[ & \left(A_{0} \cos T H_{1}-B_{0} \sin T H_{1}\right) e^{\frac{v}{2} H_{1}} \\
& \left.-\left(C_{0} \cos p_{2} H_{1}-D_{0} \sin p_{2} H_{1}\right)\right] \\
& +\varepsilon\left[\left(A_{1} \cos T H_{1}-B_{1} \sin T H_{1}\right) e^{\frac{v}{2} H_{1}}\right. \\
& \left.-\left(C_{1} \cos p_{2} H_{1}-D_{1} \sin p_{2} H_{1}\right)\right] e^{-i \eta x} d \eta \\
=\varepsilon \int_{-\infty}^{\infty}[ & \left(-T A_{0} \sin T H_{1}+\frac{v}{2} A_{0} \cos T H_{1}\right. \\
& \left.-B_{0} T \cos T H_{1}+\frac{v}{2} B_{0} \sin T H_{1}\right) e^{\frac{v}{2} H_{1}} \\
& \left.+\left(p_{2} C_{0} \sin p_{2} H_{1}+p_{2} D_{0} \cos p_{2} H_{1}\right)\right] \\
& \times h(x) e^{-i \eta x} d \eta .
\end{aligned}
$$

Now we define the Fourier transform of $h(x)$ as:

$$
\bar{h}(\lambda)=\int_{-\infty}^{\infty} h(x) e^{i \lambda x} d x
$$

and the inverse transform as:

$$
h(x)=\frac{1}{2 \pi} \int_{-\infty}^{\infty} \bar{h}(\lambda) e^{-i \lambda x} d \lambda .
$$

Therefore,

$$
h^{\prime}(x)=-\frac{i}{2 \pi} \int_{-\infty}^{\infty} \lambda \bar{h}(\lambda) e^{-i \lambda x} d \lambda .
$$

Using equations (22), (23) and (24) in equation (21), we have:

$$
\begin{aligned}
\int_{-\infty}^{\infty}[ & \left(A_{0} \cos T H_{1}-B_{0} \sin T H_{1}\right) e^{\frac{v}{2} H_{1}} \\
- & \left.\left(C_{0} \cos p_{2} H_{1}-D_{0} \sin p_{2} H_{1}\right)\right] \\
+ & \varepsilon\left[\left(A_{1} \cos T H_{1}-B_{1} \sin T H_{1}\right) e^{\frac{v}{2} H_{1}}\right. \\
& \left.-\left(C_{1} \cos p_{2} H_{1}-D_{1} \sin p_{2} H_{1}\right)\right] e^{-i \eta x} d \eta \\
= & \frac{\varepsilon}{2 \pi} \int_{-\infty}^{\infty}\left\{\int _ { - \infty } ^ { \infty } \left[\left(-T A_{0} \sin T H_{1}+\frac{v}{2} A_{0} \cos T H_{1}\right.\right.\right. \\
& \left.-B_{0} T \cos T H_{1}+\frac{v}{2} B_{0} \sin T H_{1}\right) e^{\frac{v}{2} H_{1}} \\
& \left.+\left(p_{2} C_{0} \sin p_{2} H_{1}+p_{2} D_{0} \cos p_{2} H_{1}\right)\right] \\
& \left.\times \bar{h}(\lambda) e^{-i(\eta+\lambda) x} d \eta\right\} d \lambda .
\end{aligned}
$$

Putting $\eta+\lambda=k$ for the inner integral in the right-hand side of equation (25), so that $\lambda$ may be treated as a constant such that $d \eta=d k$, replacing $\eta$ by $k$ in the left-hand side of equation (25), and finally after taking the Fourier transform as defined above, we have:

$$
\begin{aligned}
& {\left[\left(A_{0} \cos T H_{1}-B_{0} \sin T H_{1}\right) e^{\frac{v}{2} H_{1}}\right.} \\
& \left.\quad-\left(C_{0} \cos p_{2} H_{1}-D_{0} \sin p_{2} H_{1}\right)\right] \\
& \quad+\varepsilon\left[\left(A_{1} \cos T H_{1}-B_{1} \sin T H_{1}\right) e^{\frac{v}{2} H_{1}}\right. \\
& \left.\quad-\left(C_{1} \cos p_{2} H_{1}-D_{1} \sin p_{2} H_{1}\right)\right]=\varepsilon R_{1}(k),
\end{aligned}
$$

where

$$
\begin{aligned}
R_{1}(k)= & \frac{1}{2 \pi} \int_{-\infty}^{\infty}\left[\left(-T A_{0} \sin T H_{1}+\frac{v}{2} A_{0} \cos T H_{1}\right.\right. \\
& \left.-B_{0} T \cos T H_{1}+\frac{v}{2} B_{0} \sin T H_{1}\right) e^{\frac{v}{2} H_{1}} \\
& \left.+\left(p_{2} C_{0} \sin p_{2} H_{1}+p_{2} D_{0} \cos p_{2} H_{1}\right)\right] \bar{h}(\lambda) d \lambda .
\end{aligned}
$$


Using boundary condition (iii), we obtain:

$$
\begin{aligned}
\int_{-\infty}^{\infty}\{[ & \left(\mu_{2} p_{2} C_{0} \sin p_{2} H_{1}+\mu_{2} p_{2} D_{0} \cos p_{2} H_{1}\right) \\
& -\mu_{1}^{0} e^{-\frac{v}{2} H_{1}}\left(-A_{0} \frac{v}{2} \cos T H_{1}+B_{0} \frac{v}{2} \sin T H_{1}\right. \\
& \left.\left.+T A_{0} \sin T H_{1}+T B_{0} \cos T H_{1}\right)\right] \\
& +\varepsilon\left[\left(\mu_{2} p_{2} C_{1} \sin p_{2} H_{1}+\mu_{2} p_{2} D_{1} \cos p_{2} H_{1}\right)\right. \\
& -\mu_{1}^{0} e^{-\frac{v}{2} H_{1}}\left(-A_{1} \frac{v}{2} \cos T H_{1}+B_{1} \frac{v}{2} \sin T H_{1}\right. \\
& \left.\left.\left.+T A_{1} \sin T H_{1}+T B_{1} \cos T H_{1}\right)\right]\right\} e^{-i \eta x} d \eta \\
= & \int_{-\infty}^{\infty}\left\{\left(C_{0} p_{2}^{2} \mu_{2} \cos p_{2} H_{1}-D_{0} p_{2}^{2} \mu_{2} \sin p_{2} H_{1}\right)\right. \\
& +\mu_{1}^{0} e^{-\frac{v}{2} H_{1}}\left(-A_{0} \frac{v}{2} T \sin T H_{1}-B_{0} \frac{v}{2} T \cos T H_{1}\right. \\
& -T^{2} A_{0} \cos T H_{1}+T^{2} B_{0} \sin T H_{1} \\
& -\frac{v^{2}}{4} A_{0} \cos T H_{1}+\frac{v^{2}}{4} B_{0} \sin T H_{1} \\
& \left.\left.+\frac{v}{2} T A_{0} \sin T H_{1}+\frac{v}{2} T B_{0} \cos T H_{1}\right)\right\} h(x) \\
& +\left\{\left(-i \eta \mu_{2} C_{0} \cos p_{2} H_{1}+i \eta \mu_{2} D_{0} \sin p_{2} H_{1}\right)\right. \\
& \left.+\mu_{1}^{0} e^{-\frac{v}{2} H_{1}} i \eta\left(A_{0} \cos T H_{1}-B_{0} \sin T H_{1}\right)\right\} \\
& \times h^{\prime}(x) e^{-i \eta x} d \eta .
\end{aligned}
$$

Substitution of equations (22), (23) and (24) in equation (28) and proceeding as in equation (26), gives:

$$
\begin{aligned}
& {\left[\left(\mu_{2} p_{2} C_{0} \sin p_{2} H_{1}+\mu_{2} p_{2} D_{0} \cos p_{2} H_{1}\right)\right.} \\
& \quad-\mu_{1}^{0} e^{-\frac{v}{2} H_{1}}\left(-A_{0} \frac{v}{2} \cos T H_{1}+B_{0} \frac{v}{2} \sin T H_{1}\right. \\
& \left.\left.\quad+T A_{0} \sin T H_{1}+T B_{0} \cos T H_{1}\right)\right] \\
& \quad+\varepsilon\left[\left(\mu_{2} p_{2} C_{1} \sin p_{2} H_{1}+\mu_{2} p_{2} D_{1} \cos p_{2} H_{1}\right)\right. \\
& \quad-\mu_{1}^{0} e^{-\frac{v}{2} H_{1}}\left(-A_{1} \frac{v}{2} \cos T H_{1}+B_{1} \frac{v}{2} \sin T H_{1}\right. \\
& \left.\left.\quad+T A_{1} \sin T H_{1}+T B_{1} \cos T H_{1}\right)\right]=R_{2}(k),
\end{aligned}
$$

where

$$
\begin{aligned}
R_{2}(k)= & \frac{1}{2 \pi} \int_{-\infty}^{\infty}\left\{\left[\left(\mu_{2} C_{0} p_{2}^{2} \cos p_{2} H_{1}\right.\right.\right. \\
& \left.-\mu_{2} D_{0} p_{2}^{2} \sin p_{2} H_{1}\right)+\mu_{1}^{0} e^{-\frac{v}{2} H_{1}} \\
& \times\left(-A_{0} \frac{v}{2} T \sin T H_{1}-B_{0} \frac{v}{2} T \cos T H_{1}\right.
\end{aligned}
$$

$$
\begin{aligned}
& -T^{2} A_{0} \cos T H_{1}+T^{2} B_{0} \cos T H_{1} \\
& -\frac{v^{2}}{4} A_{0} \cos T H_{1}+\frac{v^{2}}{4} B_{0} \sin T H_{1} \\
& \left.\left.+\frac{v}{2} T A_{0} \sin T H_{1}+\frac{v}{2} T B_{0} \cos T H_{1}\right)\right] \\
+ & i \lambda\left[\left(-i k \mu_{2} C_{0} \cos p_{2} H_{1}+i k \mu_{2} D_{0} \sin p_{2} H_{1}\right)\right. \\
& +\mu_{1}^{0} e^{-\frac{v}{2} H_{1}} i k\left(A_{0} \cos T H_{1}\right. \\
& \left.\left.\left.-B_{0} \sin T H_{1}\right)\right]\right\} \bar{h}(\lambda) d \lambda
\end{aligned}
$$

From boundary conditions (iv) and (v), we get:

$$
\left(C_{0}+C_{1} \varepsilon\right)-\left(E+\frac{2}{p_{3}} e^{-p_{3} d}\right)=0
$$

and

$$
\left(\mu_{2} p_{2} D_{0}+\mu_{3} p_{3} E-2 \mu_{3} e^{-p_{3} d}\right)+\varepsilon \mu_{2} p_{2} D_{1}=0,(32
$$

respectively. Equating the absolute terms (i.e., the terms not containing $\varepsilon$ ) and the coefficient of $\varepsilon$ from equations (20), (26), (29), (31) and (32), we get:

$$
\begin{aligned}
& A_{0} T \sin T H_{2}+B_{0} T \cos T H_{2}-\frac{v}{2} A_{0} \cos T H_{2} \\
& \quad+\frac{v}{2} B_{0} \sin T H_{2}=0,
\end{aligned}
$$

$A_{1} T \sin T H_{2}+B_{1} T \cos T H_{2}-\frac{v}{2} A_{1} \cos T H_{2}$

$$
+\frac{v}{2} B_{1} \sin T H_{2}=0
$$

$$
\begin{aligned}
& \left(A_{0} \cos T H_{1}-B_{0} \sin T H_{1}\right) e^{\frac{v}{2} H_{1}} \\
& \quad-\left(C_{0} \cos p_{2} H_{1}-D_{0} \sin p_{2} H_{1}\right)=0
\end{aligned}
$$

$$
\begin{aligned}
& \left(A_{1} \cos T H_{1}-B_{1} \sin T H_{1}\right) e^{\frac{v}{2} H_{1}} \\
& \quad-\left(C_{1} \cos p_{2} H_{1}-D_{1} \sin p_{2} H_{1}\right)=R_{1},
\end{aligned}
$$

$\left(\mu_{2} p_{2} C_{0} \sin p_{2} H_{1}+\mu_{2} p_{2} D_{0} \cos p_{2} H_{1}\right)-\mu_{1}^{0} e^{-\frac{v}{2} H_{1}}$

$$
\begin{aligned}
& \times\left(-A_{0} \frac{v}{2} \cos T H_{1}+B_{0} \frac{v}{2} \sin T H_{1}\right. \\
& \left.+T A_{0} \sin T H_{1}+T B_{0} \cos T H_{1}\right)=0,
\end{aligned}
$$

$$
\begin{aligned}
& \left(\mu_{2} p_{2} C_{1} \sin p_{2} H_{1}+\mu_{2} p_{2} D_{1} \cos p_{2} H_{1}\right)-\mu_{1}^{0} e^{-\frac{v}{2} H_{1}} \\
& \quad \times\left(-A_{1} \frac{v}{2} \cos T H_{1}+B_{1} \frac{v}{2} \sin T H_{1}\right. \\
& \left.\quad+T A_{1} \sin T H_{1}+T B_{1} \cos T H_{1}\right)=R_{2},
\end{aligned}
$$




$$
\begin{gathered}
C_{0}-E-\frac{2}{p_{3}} E^{-p_{3} d}=0, \\
C_{1}=0, \quad D_{1}=0, \\
p_{2} \mu_{2} D_{0}+\mu_{3} p_{3} E-2 \mu_{3} e^{-p_{3} d}=0 .
\end{gathered}
$$

Solving the above equations, expressions for the arbitrary constants can be easily deduced. The values of arbitrary constants are provided in Appendix I.

The displacement in the uppermost layer is given by:

$V_{1}=\frac{1}{2 \pi} \int_{-\infty}^{\infty} \frac{2 \mu_{2} p_{2} \mu_{3} e^{-p_{3} d}\left(2 T \tan T H_{2}-v\right)}{U(k)}$

$\times\left\{1+\frac{\varepsilon\left[\begin{array}{c}R_{1} \mu_{1}^{0}\left(-v \cos T H_{1}+2 T \sin T H_{1}\right) e^{-\frac{v}{2} H_{1}} \\ +2 R_{2} \cos T H_{1} e^{\frac{v}{2} H_{1}}\end{array}\right]}{4 \mu_{2} p_{2} \mu_{3} \mu_{1}^{0} T\left(2 T \tan T H_{2}-v\right)}\right.$

$\left.\times e^{p_{3} d} U(k)\right\}\left[-\frac{\left(2 T+v \tan T H_{2}\right)}{\left(2 T \tan T H_{2}-v\right)} \cos T z+\sin T z\right]$

$\times e^{-\frac{v}{2} z} e^{-i \eta x} d \eta$

where $U(k)=Q_{1}+Q_{2} \tan T H_{2} \cdot Q_{1}$ and $Q_{2}$ are provided in Appendix I.

\subsection{Case I}

When the interface of uppermost layer and intermediate layer contains the irregularity of rectangular shape of depth $H^{\prime}$ and span $2 s$ :

Taking the Fourier transform of equation (2), we get:

$$
\bar{h}(\lambda)=\frac{4 s}{\lambda} \sin (\lambda s)
$$

Using equations (27) and (30), we get:

$\frac{1}{2} R_{1} \mu_{1}^{0}\left(-v \cos T H_{1}+2 T \sin T H_{1}\right) e^{-\frac{v}{2} H_{1}}$

$$
\begin{aligned}
& +R_{2} \cos T H_{1} e^{\frac{v}{2} H_{1}} \\
& =\frac{2 s}{\pi} \int_{-\infty}^{\infty}[\psi(k-\lambda)+\psi(k+\lambda)] \frac{1}{\lambda} \sin (\lambda s) d \lambda,
\end{aligned}
$$

where

$$
\psi(k-\lambda)=\left[A_{2}+A_{3}+A_{4}\right]^{\eta=k-\lambda} \frac{e^{-p_{3} d}}{U(k)}
$$

with

$$
\begin{aligned}
& A_{2}=Q_{3}+Q_{4} \tan T H_{2}, \\
& A_{3}=Q_{5}+Q_{6} \tan T H_{2}, \\
& A_{4}=Q_{7}+Q_{8} \tan T H_{2},
\end{aligned}
$$

where $Q_{3}, Q_{4}, Q_{5}, Q_{6}, Q_{7}$ and $Q_{8}$ are provided in Appendix.

Here the argument of $\psi(k-\lambda)$ is because of $\eta+$ $\lambda=k$.

Using asymptotic formula of Willis (1948) and neglecting the terms containing $2 / s$ and higher power of $2 / s$ for large $s$, we have (Tranter 1966)

$$
\begin{aligned}
\int_{-\infty}^{\infty}[ & \psi(k-\lambda)+\psi(k+\lambda)] \frac{1}{\lambda} \sin \frac{\lambda s}{2} d \lambda \\
& \cong \frac{\pi}{2} 2 \psi(k) \\
& =\pi \psi(k) .
\end{aligned}
$$

Using equation (49) in equation (44), we obtain $\frac{1}{2} R_{1} \mu_{1}^{0}\left(-v \cos T H_{1}+2 T \sin T H_{1}\right)+R_{2} \cos T H_{1} e^{\frac{v}{2} H_{1}}$

$$
=2 s \psi(k)=\frac{H^{\prime}}{\varepsilon} \psi(k) .
$$

Therefore, the displacement in the uppermost layer is:

$$
\begin{aligned}
V_{1}= & \frac{1}{2 \pi} \int_{-\infty}^{\infty} \frac{2 \mu_{2} p_{2} \mu_{3} e^{-p_{3} d}\left(2 T \tan T H_{2}-v\right)}{U(k)\left[1-\left(H^{\prime} \psi(k) e^{p_{3} d} U(k) / 2 \mu_{2} p_{2} T \mu_{1}^{0} \mu_{3}\left(2 T \tan T H_{2}-v\right)\left(\mu_{2} p_{2} \cos p_{2} H_{1}+\mu_{3} p_{3} \sin p_{2} H_{1}\right)\right)\right]} \\
& \times\left[-\frac{\left(2 T+v \tan T H_{2}\right)}{\left(2 T \tan T H_{2}-v\right)} \cos T z+\sin T z\right] e^{-\frac{v}{2} z} e^{-i \eta x} d \eta
\end{aligned}
$$


The value of above integral depends entirely on the contribution of the poles of the integrand. The poles are located at the roots of equation

$$
U(k)\left[1-\frac{H^{\prime} \psi(k) e^{p_{3} d} U(k)}{\left[\begin{array}{c}
2 \mu_{2} p_{2} T \mu_{1}^{0} \mu_{3}\left(2 T \tan T H_{2}-v\right) \\
\times\left(\mu_{2} p_{2} \cos p_{2} H_{1}+\mu_{3} p_{3} \sin p_{2} H_{1}\right)
\end{array}\right]}\right]=0 .
$$

Now, on simplification equation (52) leads to

$$
S_{1} \tan ^{2} T H_{2}+S_{2} \tan T H_{2}+S_{3}=0,
$$

where $S_{1}, S_{2}, S_{3}$ are provided in Appendix I.

If $c$ is the common wave velocity of the wave propagating along the surface, then one can define (where $\omega=c k$ and $k$ is the wave number),

$$
P_{2}=\frac{p_{2}}{k}, \quad P_{3}=\frac{p_{3}}{k} \quad \text { and } \quad \mathrm{t}=\frac{T}{k},
$$

where

$$
\begin{aligned}
& P_{2}=\sqrt{\frac{c^{2}}{\beta_{2}^{2}}-1-\xi^{\prime}}, \quad P_{3}=\sqrt{1-\xi^{\prime}-\frac{c^{2}}{\beta_{3}^{2}}}, \\
& t=\frac{1}{2} \sqrt{\frac{-v^{2}}{k^{2}}+4\left(1+\xi-\frac{c^{2}}{\beta_{1}^{2}}\right)} .
\end{aligned}
$$

In view of equations (54) and (55), equation (53) gives

$$
S_{4} \tan ^{2} t K H_{2}+S_{5} \tan t K H_{2}+S_{6}=0,
$$

where $S_{4}, S_{5}$ and $S_{6}$ are given in Appendix.

Equation (56) is the dispersion equation for the propagation of Love-type wave in an initially stressed vertically heterogeneous irregular isotropic layer with rectangular irregularity lying over an initially stressed isotropic layer and initially stressed isotropic half-space.

\subsection{Case II}

When the interface of uppermost layer and intermediate layer contains the irregularity of parabolic shape of maximum depth $H^{\prime}$ and span $2 s$ :

Taking the Fourier transform of equation (3), we get:

$$
\bar{h}(\lambda)=\frac{4 H^{\prime} s}{\varepsilon} \frac{\sin (\lambda s)-\lambda s \cos (\lambda s)}{(\lambda s)^{3}} .
$$

Using equations (27) and (30), we get:

$$
\begin{gathered}
R_{1} \mu_{1}^{0}\left(-\frac{v}{2} \cos T H_{1}+T \sin T H_{1}\right) e^{-\frac{v}{2} H_{1}}+R_{2} \cos T H_{1} e^{\frac{v}{2} H_{1}} \\
=\frac{2 H^{\prime} s}{\pi \varepsilon} \int_{0}^{\infty}[\psi(k-\lambda)+\psi(k+\lambda)] \sqrt{\frac{\pi}{2}} \frac{J_{3 / 2}(\lambda s)}{(\lambda s)^{3 / 2}} d \lambda
\end{gathered}
$$

where $J_{3 / 2}(\lambda s)$ is a Bessel function of the first kind of order $3 / 2$.

Using asymptotic formula of Willis (1948) and neglecting the terms containing $2 / s$ and higher power of $2 / s$ for large $s$, we have (Tranter 1966):

$$
\int_{0}^{\infty}[\psi(k-\lambda)+\psi(k+\lambda)] \sqrt{\frac{\pi}{2}} \frac{J_{3 / 2}(\lambda s)}{(\lambda s)^{3 / 2}} d \lambda \cong \frac{2}{3 s} \psi(k) .
$$

Using equation (59) in equation (58), we obtain:

$$
\begin{gathered}
\frac{1}{2} R_{1} \mu_{1}^{0}\left(-v \cos T H_{1}+2 T \sin T H_{1}\right)+R_{2} \cos T H_{1} e^{\frac{v}{2} H_{1}} \\
=\frac{2 H^{\prime} s}{\pi \varepsilon} \cdot \frac{2}{3 s} \psi(k)=\frac{4 H^{\prime}}{3 \pi \varepsilon} \psi(k) .
\end{gathered}
$$

Therefore, the displacement in the uppermost layer is:

$$
\begin{aligned}
V_{1}= & \frac{1}{2 \pi} \int_{-\infty}^{\infty} \frac{2 \mu_{2} p_{2} \mu_{3} e^{-p_{3} d}\left(2 T \tan T H_{2}-v\right)}{U(k)\left[1-\left(H^{\prime \prime} \psi(k) e^{p_{3} d} U(k) / 2 \mu_{2} p_{2} T \mu_{1}^{0} \mu_{3}\left(2 T \tan T H_{2}-v\right)\left(\mu_{2} p_{2} \cos p_{2} H_{1}+\mu_{3} p_{3} \sin p_{2} H_{1}\right)\right)\right]} \\
& \times\left[-\frac{\left(2 T+v \tan T H_{2}\right)}{\left(2 T \tan T H_{2}-v\right)} \cos T z+\sin T z\right] e^{-\frac{v}{2} z} e^{-i \eta x} d \eta
\end{aligned}
$$

where $H^{\prime \prime}=\frac{4}{3 \pi} H^{\prime}$.

The value of above integral depends entirely on the contribution of the poles of the integrand. The poles are located at the roots of equation

$$
U(k)\left[1-\frac{H^{\prime \prime} \psi(k) e^{p_{3} d} U(k)}{2 \mu_{2} p_{2} T \mu_{1}^{0} \mu_{3}\left(2 T \tan T H_{2}-v\right)\left(\mu_{2} p_{2} \cos p_{2} H_{1}+\mu_{3} p_{3} \sin p_{2} H_{1}\right)}\right]=0
$$


Equation (62) after simplification gives:

$$
F_{1} \tan ^{2} T H_{2}+F_{2} \tan T H_{2}+F_{3}=0
$$

where $F_{1}, F_{2}, F_{3}$ are provided in Appendix I.

Using equations (54) and (55), equation (63) leads to

$$
F_{4} \tan ^{2} t k H_{2}+F_{5} \tan t k H_{2}+F_{6}=0,
$$

where $F_{4}, F_{5}$ and $F_{6}$ are given in Appendix.

Equation (64) is the dispersion equation for the propagation of Love-type wave in an initially stressed vertically heterogeneous irregular isotropic layer with parabolic irregularity lying over an initially stressed isotropic layer and initially stressed isotropic half-space.

\section{Particular cases}

\subsection{Case 1}

When $v=0$ and $S_{11}^{(0)}=0$, equations (56) and (64) reduces to

$$
S_{4}^{(1)} \tan ^{2} t^{(1)} k H_{2}+S_{5}^{(1)} \tan t^{(1)} k H_{2}+S_{6}^{(1)}=0
$$

and

$$
F_{4}^{(1)} \tan ^{2} t^{(1)} k H_{2}+F_{5}^{(1)} \tan t^{(1)} k H_{2}+F_{6}^{(1)}=0
$$

respectively. $S_{4}^{(1)}, S_{5}^{(1)}, S_{6}^{(1)}, t^{(1)}, F_{4}^{(1)}, F_{5}^{(1)}, F_{6}^{(1)}$ are provided in Appendix. Equations (65) and (66) are the dispersion relation for the propagation of Love-type wave in an irregular isotropic homogeneous layer without initial stress lying over an initially stressed isotropic layer and an initially stressed isotropic half-space when irregularity is considered in rectangular form and parabolic form respectively.

\subsection{Case 2}

When $v=0, S_{11}^{(0)}=0, S_{11}^{(2)}=0$ and $S_{11}^{(3)}=0$, equations (56) and (64) takes the form

$$
S_{4}^{(2)} \tan ^{2} t^{(1)} k H_{2}+S_{5}^{(2)} \tan t^{(1)} k H_{2}+S_{6}^{(2)}=0
$$

and

$$
F_{4}^{(2)} \tan ^{2} t^{(1)} k H_{2}+F_{5}^{(2)} \tan t^{(1)} k H_{2}+F_{6}^{(2)}=0
$$

respectively. $S_{4}^{(2)}, S_{5}^{(2)}, S_{6}^{(2)}, F_{4}^{(2)}, F_{5}^{(2)}, F_{6}^{(2)}$ are provided in Appendix. Equations (67) and (68) are the dispersion equation for the propagation of Love-type wave in an irregular isotropic homogeneous layer without initial stress lying over an isotropic layer and an isotropic half-space without initial stress when irregularity is considered in rectangular form and parabolic form respectively.

\subsection{Case 3}

When $v=0, S_{11}^{(2)}=0, S_{11}^{(3)}=0, H^{\prime \prime}=0$, and $H^{\prime}=0$, both equations (56) and (64) lead to

$$
\begin{aligned}
& \tan t^{(2)} k H_{2} \\
&= \frac{\left[\begin{array}{c}
-\mu_{2} P_{2}^{(1)}\left(\mu_{2} P_{2}^{(1)} \tan P_{2}^{(1)} k H_{1}-\mu_{3} P_{3}^{(1)}\right) \\
+\mu_{1}^{0} t^{(2)} \tan t^{(2)} k H_{1}\left(\mu_{2} P_{2}^{(1)}\right.
\end{array}\right]}{\left.-\mu_{3} P_{3}^{(1)} \tan P_{2}^{(1)} k H_{1}\right)} \\
& {\left[\begin{array}{c}
\mu_{2} P_{2}^{(1)} \tan t^{(2)} k H_{1} \\
\times\left(\mu_{2} P_{2}^{(1)} \tan P_{2}^{(1)} k H_{1}-\mu_{3} P_{3}^{(1)}\right) \\
+\mu_{1}^{0} t^{(2)}\left(\mu_{2} P_{2}^{(1)}+\mu_{3} P_{3}^{(1)} \tan P_{2}^{(1)} k H_{1}\right)
\end{array}\right] }
\end{aligned}
$$

which is the dispersion equation for the propagation of Love-type wave in a regular initially stressed isotropic homogeneous layer without initial stress lying over an isotropic layer and an isotropic half-space without initial stress. $t^{(2)}, P_{2}^{(1)}, P_{3}^{(1)}$ are provided in Appendix.

\subsection{Case 4}

When $v=0, S_{11}^{(0)}=0, S_{11}^{(2)}=0, S_{11}^{(3)}=0, H^{\prime}=0$, $H^{\prime \prime}=0$, both equations (56) and (64) reduces to

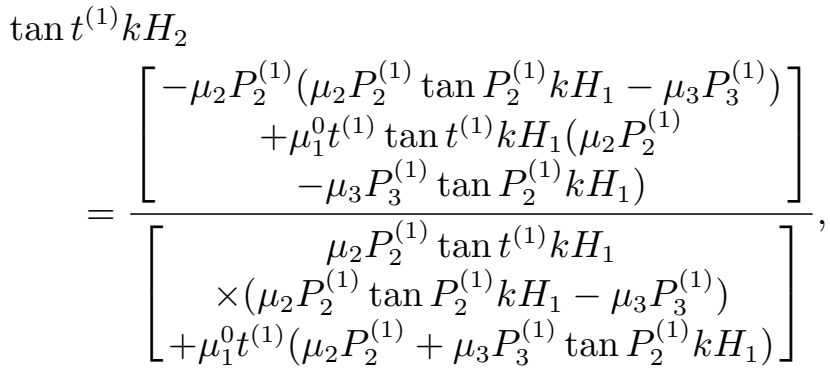

which is the dispersion equation for the propagation of Love-type wave in a regular isotropic homogeneous layer without initial stress lying over an isotropic layer and an isotropic half-space without initial stress.

\subsection{Case 5}

When $v=0, S_{11}^{(0)}=0, S_{11}^{(2)}=0, S_{11}^{(3)}=0, H^{\prime}=0$, $H^{\prime \prime}=0, H_{2} \rightarrow H_{1}$, both equations (56) and (64) becomes

$$
\tan k H \sqrt{\frac{c^{2}}{\beta_{2}^{2}}-1}=\frac{\mu_{3} \sqrt{1-c^{2} / \beta_{3}^{2}}}{\mu_{2} \sqrt{c^{2} / \beta_{2}^{2}-1}},
$$

which is the classical Love-wave equation. 


\section{Numerical results and discussion}

The following data has been considered for numerical computation of phase velocity of Love-type wave propagating in an initially stressed irregular heterogeneous isotropic layer lying over an initially stressed isotropic layer and an initially stressed isotropic half-space (Gubbins 1990):

For uppermost layer:

$$
\begin{aligned}
& \mu_{1}=32.3 \times 10^{9} \mathrm{~N} / \mathrm{m}^{2}, \\
& \rho_{1}=2803 \mathrm{~kg} / \mathrm{m}^{3} .
\end{aligned}
$$

For intermediate layer:

$$
\begin{aligned}
& \mu_{2}=65.4 \times 10^{9} \mathrm{~N} / \mathrm{m}^{2}, \\
& \rho_{2}=3409 \mathrm{~kg} / \mathrm{m}^{3} .
\end{aligned}
$$

For lowermost half-space:

$$
\begin{aligned}
\mu_{3} & =291.7 \times 10^{9} \mathrm{~N} / \mathrm{m}^{2}, \\
\rho_{3} & =5563 \mathrm{~kg} / \mathrm{m}^{3} .
\end{aligned}
$$

Moreover, we consider the following data:

$$
\begin{aligned}
& v H_{1}=0.01,0.03,0.05,1.1 \\
& H^{\prime} / H_{1}=0.1,0.3,0.5 \\
& H_{2} / H_{1}=1.1,1.5,2.5,3.5 \\
& \xi_{1}=0, \pm 0.2, \pm 0.4 \\
& \xi_{2}=0, \pm 0.05, \pm 0.1,0.2 \\
& \xi_{3}=0, \pm 0.2, \pm 0.4
\end{aligned}
$$

Graphical interpretation of dispersion curve reflecting the effect of different affecting parameters, viz., heterogeneity $\left(\nu H_{1}\right)$, irregularity $\left(H^{\prime} / H_{1}\right)$, width ratio of layers $\left(H_{2} / H_{1}\right)$, horizontal initial stress acting in uppermost layer $\left(\xi_{1}\right)$, horizontal initial stress acting in sandwiched layer $\left(\xi_{2}\right)$ and horizontal initial stress acting in lowermost half-space $\left(\xi_{3}\right)$, have been shown in figures $3-8$. All the figures show the variation of phase velocity of Love-type wave against wave number for different values of affecting parameters. It can be easily perceived from all these figures that the phase velocity of Love-type wave decreases with increase in wave number. To perform a comparative study for the case of rectangular irregularity with that of parabolic irregularity existing at the interface of uppermost layer and intermediate layer, we carried out numerical computations and depicted them graphically in figures $3-8$. In each of these figures, solid line curves correspond to the case of rectangular irregularity and dotted line curves correspond to the case of parabolic irregularity.

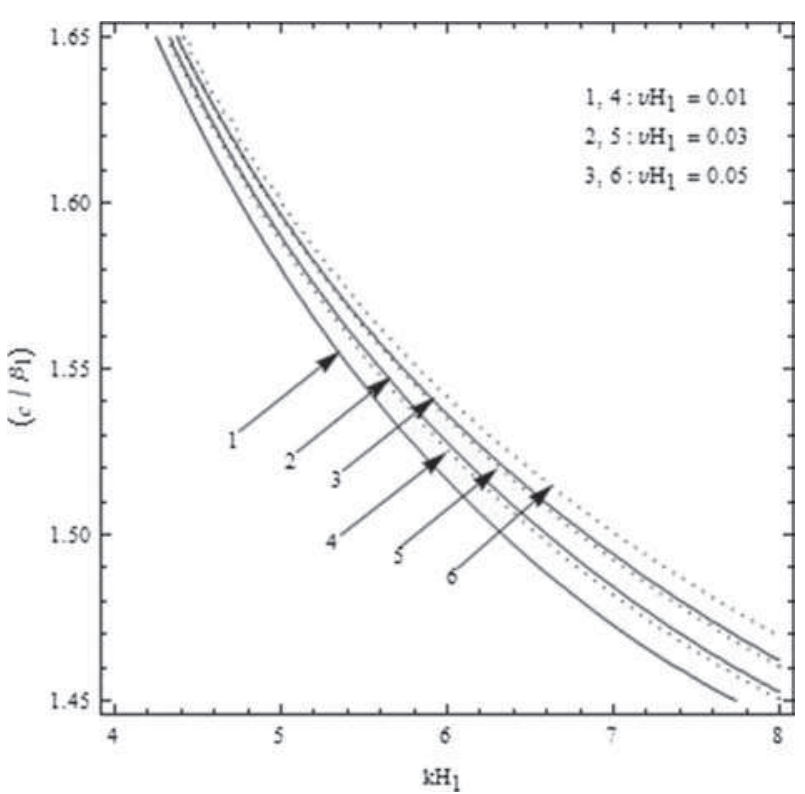

Figure 3. Phase velocity $\left(c / \beta_{1}\right)$ against wave number $\left(k H_{1}\right)$ for different values of heterogeneity parameter $\left(v H_{1}\right)$ when $\xi_{1}=0.2, \xi_{2}=0.2, \xi_{3}=0.2, H^{\prime} / H_{1}=0.1, H_{2} / H_{1}=1.1$.

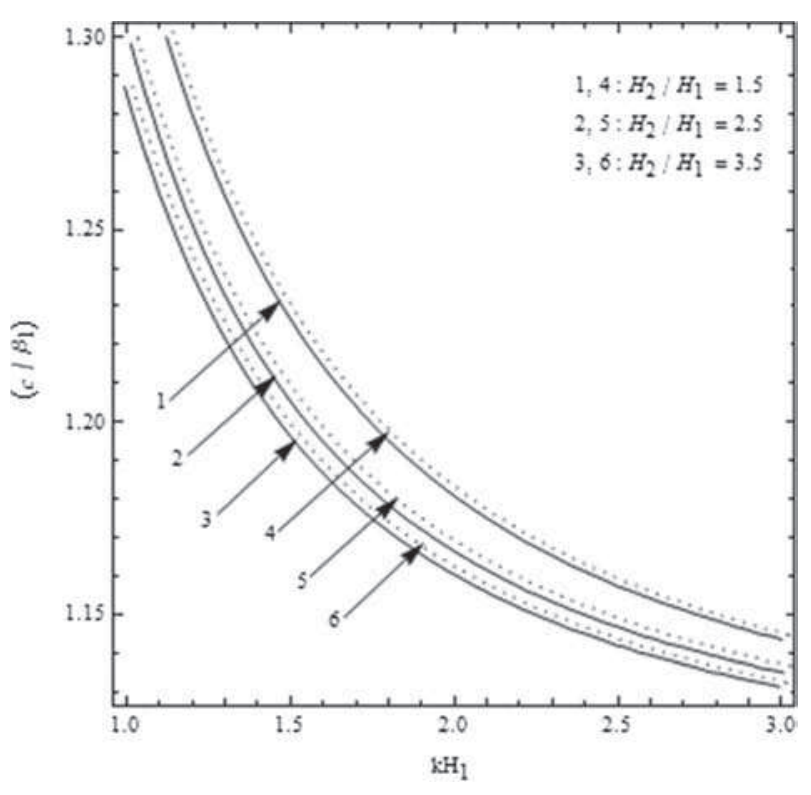

Figure 4 . Phase velocity $\left(c / \beta_{1}\right)$ against wave number $\left(k H_{1}\right)$ for different values of width ratio of layers $\left(H_{2} / H_{1}\right)$ when $\xi_{1}=0.2, \xi_{2}=0.2, \xi_{3}=0.2, H^{\prime} / H_{1}=0.1, v H_{1}=1.1$.

Figure 3 enlightens the effect of heterogeneity acting in the uppermost layer on the phase velocity of Love-type wave for both the cases when irregularity is considered in rectangular form and parabolic form. The figure particularizes that dispersion curve shifts upward as the heterogeneity increases, i.e., the phase velocity increases as heterogeneity 


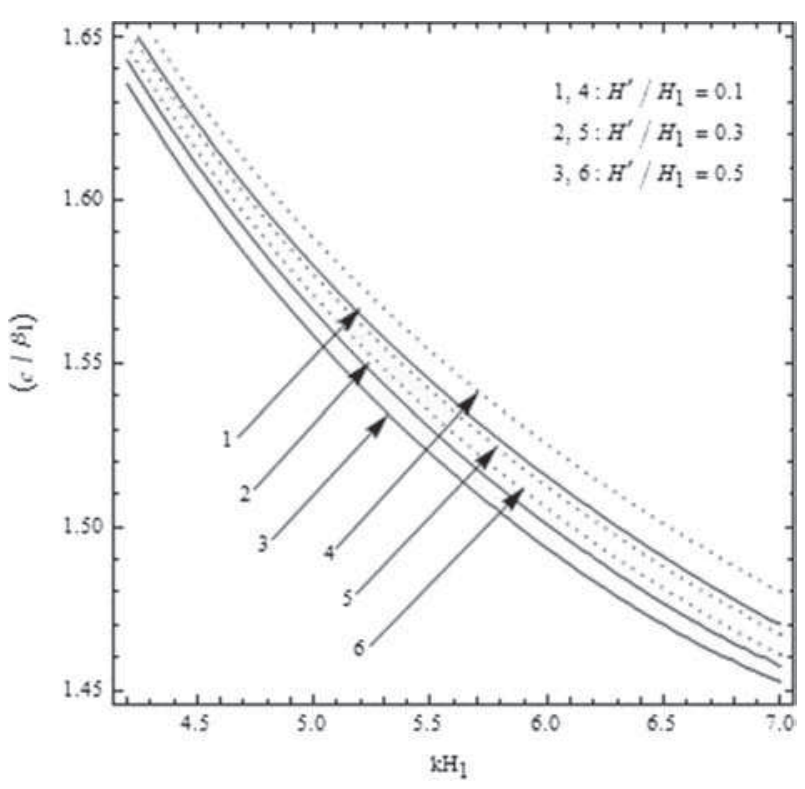

Figure 5. Phase velocity $\left(c / \beta_{1}\right)$ against wave number $\left(k H_{1}\right)$ for different values of irregularity parameter $H^{\prime} / H_{1}$ when $\xi_{1}=0.2, \xi_{2}=0.2, \xi_{3}=0.2, v H_{1}=0.01, H_{2} / H_{1}=1.5$.

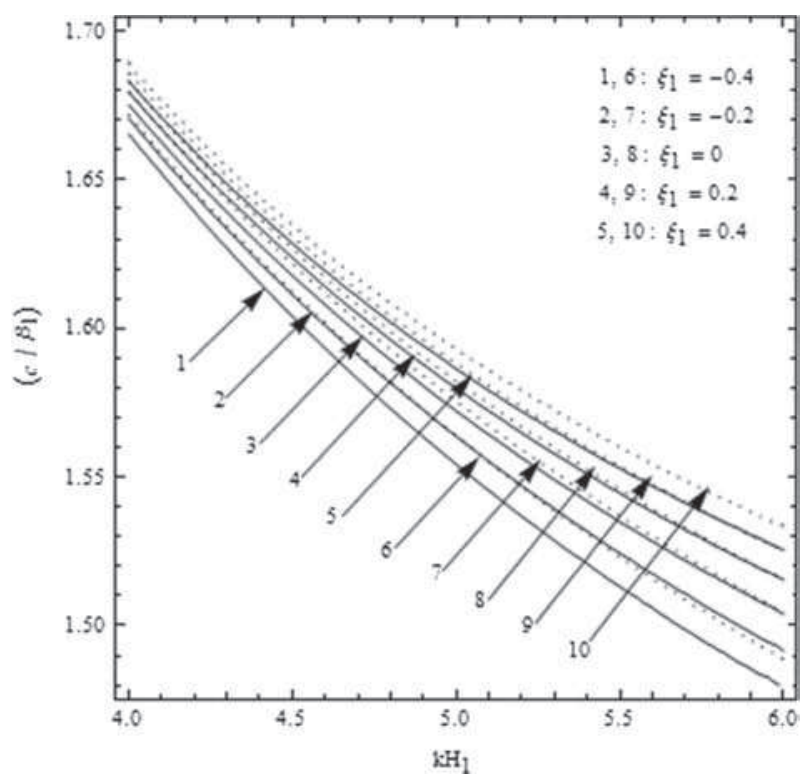

Figure 6 . Phase velocity $\left(c / \beta_{1}\right)$ against wave number $\left(k H_{1}\right)$ for different values of horizontal initial stress acting in the uppermost layer $\left(\xi_{1}\right)$ when $\xi_{2}=0.2, \xi_{3}=0.2, H^{\prime} / H_{1}=0.1$, $v H_{1}=0.01, H_{2} / H_{1}=1.5$.

grows in the uppermost layer. Moreover, minute examination of the curves adduce that the effect of heterogeneity on phase velocity of Love-type wave is less at low frequency region as compared to high frequency region.

The effect of width ratio of layers on the phase velocity of Love-type wave has been shown in figure 4. It has been noticed from the figure that as the width ratio increases dispersion curve shifts downwards, i.e., phase velocity decreases

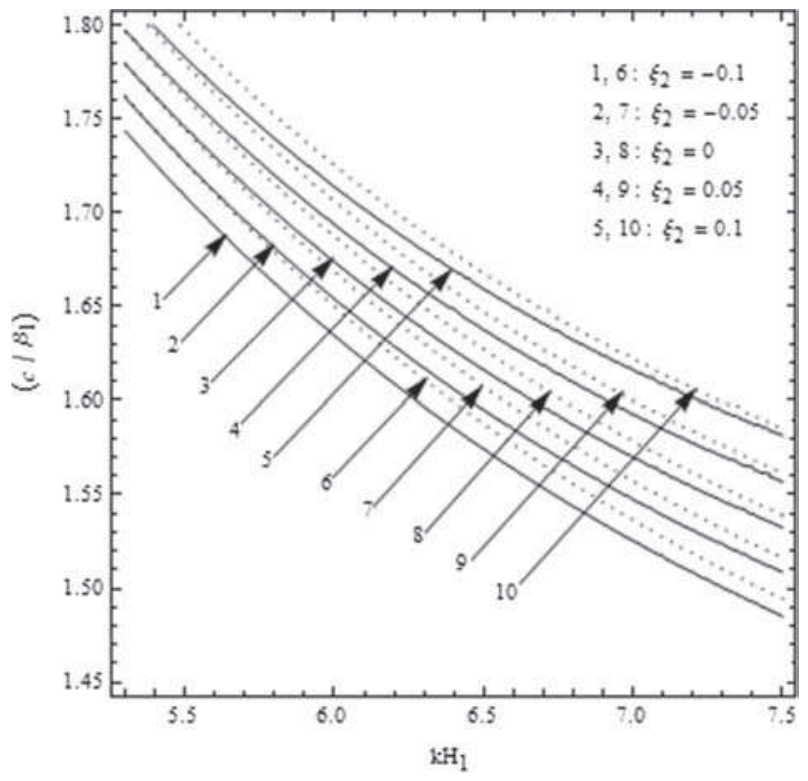

Figure 7 . Phase velocity $\left(c / \beta_{1}\right)$ against wave number $\left(k H_{1}\right)$ for different values of horizontal initial stress acting in the intermediate layer $\left(\xi_{2}\right)$ when $\xi_{1}=0.2, \xi_{3}=0.2, H^{\prime} / H_{1}=0.1$, $v H_{1}=0.01, H_{2} / H_{1}=1.5$.

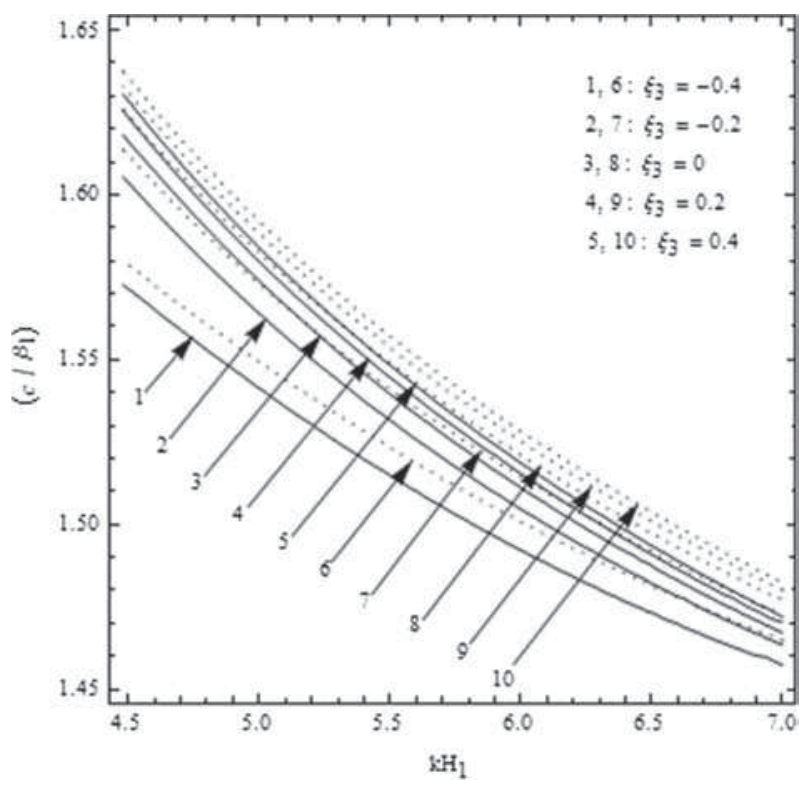

Figure 8 . Phase velocity $\left(c / \beta_{1}\right)$ against wave number $\left(k H_{1}\right)$ for different values of horizontal initial stress acting in the half-space $\left(\xi_{3}\right)$ when $\xi_{1}=0.2, \xi_{2}=0.2, H^{\prime} / H_{1}=0.1$, $v H_{1}=0.01, H_{2} / H_{1}=1.5$.

with increase in width ratio of layers for both the cases when the irregularity is considered in rectangular and parabolic form existing at the interface of uppermost layer and intermediate layer. Also, it can be concluded that with increase in the magnitude of width ratio, its effect on the phase velocity of Love-type wave decreases for both rectangular and parabolic irregularity cases. 
Figure 5 delineates the effect of irregularity parameter on the phase velocity of Love-type wave. It is evident from the figure that phase velocity of Love-type wave decreases with increase in size of irregularity for both the case of rectangular and parabolic irregularity. As like the effect of width ratio on phase velocity, figure 5 elucidates that with increase in the magnitude of irregularity, its effect on phase velocity of Love-type wave declines for both rectangular and parabolic irregularity cases.

Figures 6, 7 and 8 render the effect of horizontal initial stress associated with the uppermost layer, intermediate layer and lowermost half-space, respectively, on the phase velocity of Love-type wave for both the cases of rectangular irregularity and parabolic irregularity existing at the interface of uppermost layer and intermediate layer. It has been deduced from the figures that phase velocity increases with the increase in horizontal initial stresses acting in uppermost layer, intermediate layer and lowermost half-space for both the cases of rectangular irregularity and parabolic irregularity. More precisely, in each of the three mediums, phase velocity increases as the horizontal compressive initial stress increases whereas phase velocity decreases as the horizontal tensile initial stress increases. In particular, figure 8 illustrates that the effect of horizontal tensile initial stress on the phase velocity of Love-type wave is comparatively much pronounced than the horizontal compressive initial stress associated with lowermost half-space.

A meticulous observation specifies that although each of the initial stresses acting in the medium portray similar behaviour on the dispersion curve yet the initial stress associated with the intermediate layer affects the dispersion curve the most in comparison to the initial stresses associated with uppermost layer and lowermost half-space. The

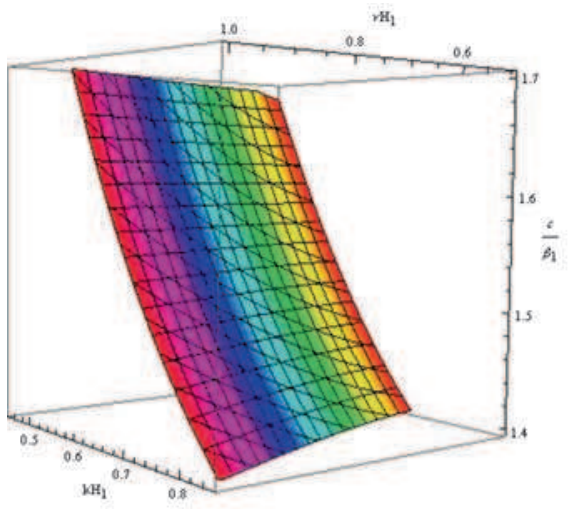

(i)

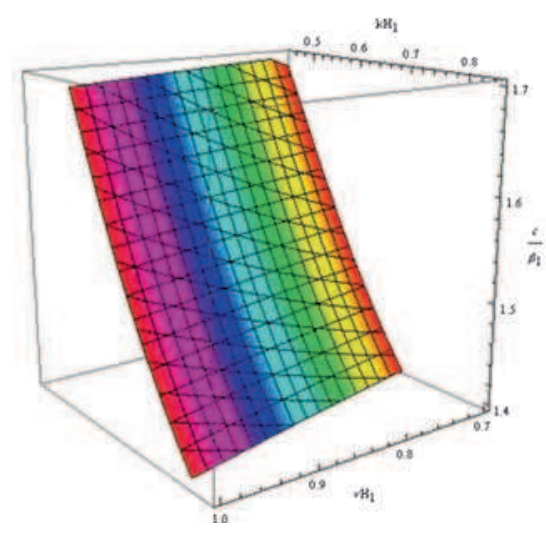

(ii)

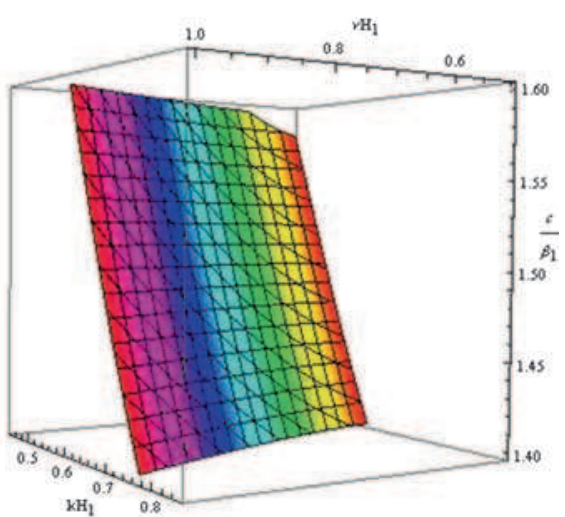

(iii)

Figure 9. Phase velocity $\left(c / \beta_{1}\right)$ against wave number $\left(k H_{1}\right)$ and heterogeneity parameter $\left(v H_{1}\right)$ when rectangular irregularity is considered and $H_{2} / H_{1}=1.5, H^{\prime} / H_{1}=0.1$; for (i) $\xi_{1}=0.1, \xi_{2}=0.1, \xi_{3}=0.1$, (ii) $\xi_{1}=0, \xi_{2}=0, \xi_{3}=0$; (iii) $\xi_{1}=-0.1, \xi_{2}=-0.1, \xi_{3}=-0.1$.

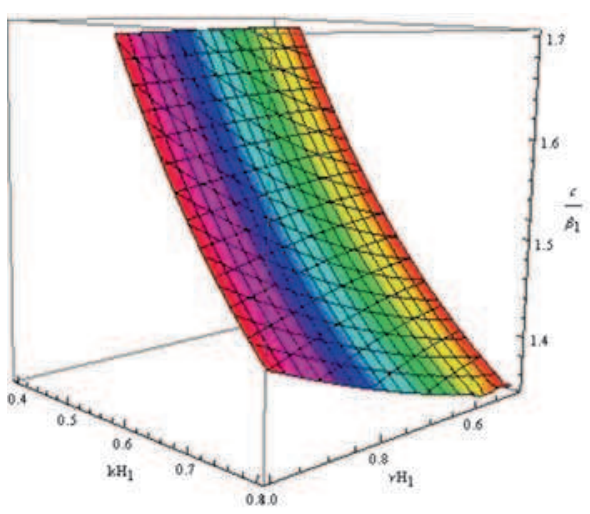

(i)

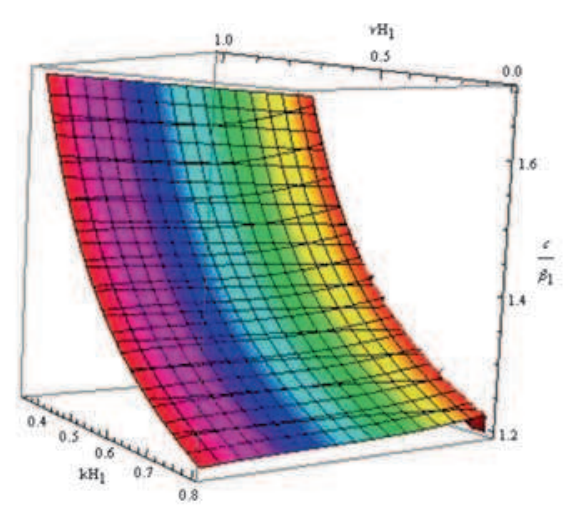

(ii)

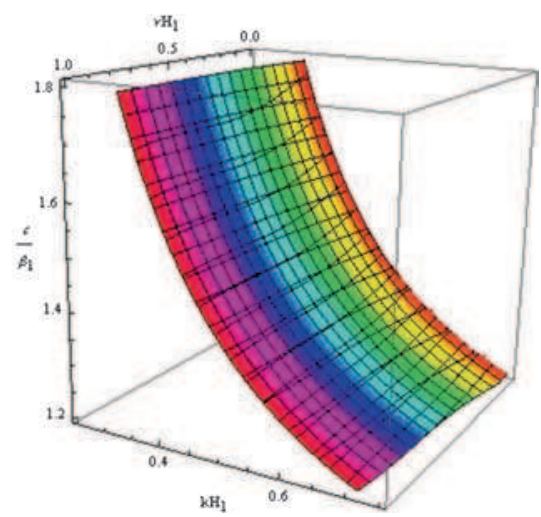

(iii)

Figure 10. Phase velocity $\left(c / \beta_{1}\right)$ against wave number $\left(k H_{1}\right)$ and heterogeneity parameter $\left(v H_{1}\right)$ when parabolic irregularity is considered and $H_{2} / H_{1}=1.5, H^{\prime} / H_{1}=0.1$; for (i) $\xi_{1}=0.1, \xi_{2}=0.1, \xi_{3}=0.1$, (ii) $\xi_{1}=0, \xi_{2}=0, \xi_{3}=0$; (iii) $\xi_{1}=-0.1, \xi_{2}=-0.1, \xi_{3}=-0.1$. 
effect of initial stresses acting in the uppermost layer and intermediate layer on the phase velocity of Love-type wave is less in the low frequency region but significant at high frequency region for both the cases of rectangular irregularity and parabolic irregularity. The effect of initial stress acting in lowermost half-space on the phase velocity of Lovetype wave is significant in the low frequency region whereas it is comparatively less at high frequency region for both the cases of rectangular irregularity and parabolic irregularity.

An overview and comparative study of all the curves in figures 3-8 signify that the dotted line curves always lie above the solid line curves, i.e., the curve corresponding to parabolic irregularity dominates the curve corresponding to rectangular irregularity. Therefore, these figures reveal that parabolic irregularity supports comparatively more phase velocity than the rectangular irregularity present at the interface of uppermost layer and intermediate layer.

The variation of dimensionless phase velocity against dimensionless wave number and heterogeneity associated with uppermost layer are shown by means of surface plots in figures 9 and 10 for the case of rectangular irregularity and parabolic irregularity respectively persisting at the interface of uppermost layer and intermediate layer. Surface plots in figures 11 and 12 irradiate the variation of dimensionless phase velocity against dimensionless wave number and irregularity for the case of rectangular irregularity and parabolic irregularity

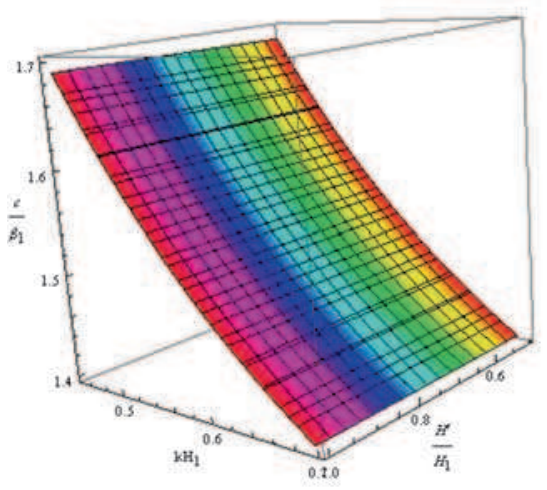

(i)

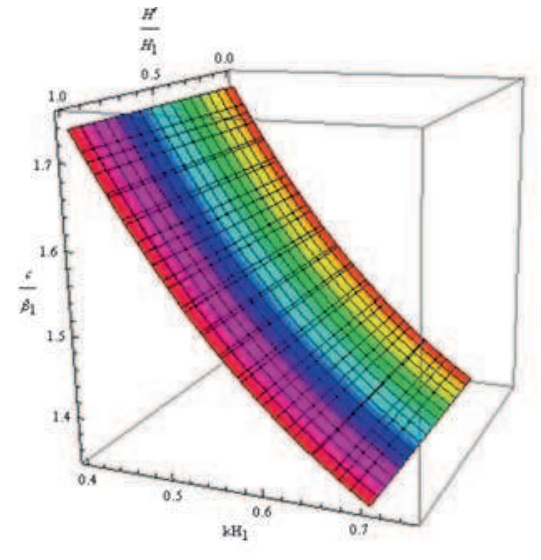

(ii)

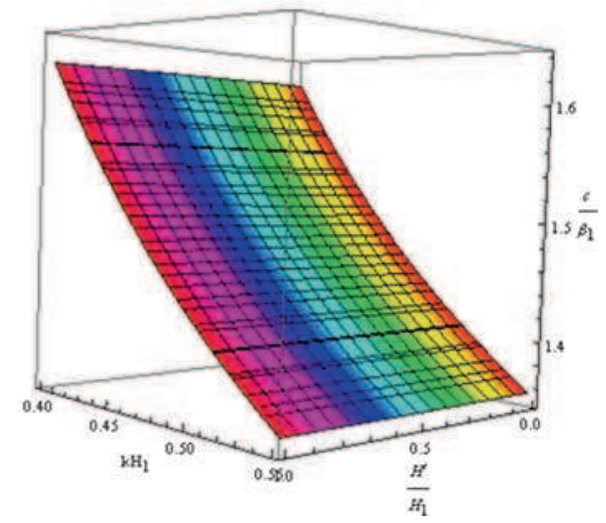

(iii)

Figure 11. Phase velocity $\left(c / \beta_{1}\right)$ against wave number $\left(k H_{1}\right)$ and irregularity parameter $\left(H^{\prime} / H_{1}\right)$ when rectangular irregularity is considered and $H_{2} / H_{1}=1.5, v H_{1}=0.1$; for (i) $\xi_{1}=0.1, \xi_{2}=0.1, \xi_{3}=0.1$, (ii) $\xi_{1}=0, \xi_{2}=0, \xi_{3}=0$; (iii) $\xi_{1}=-0.1, \xi_{2}=-0.1, \xi_{3}=-0.1$.

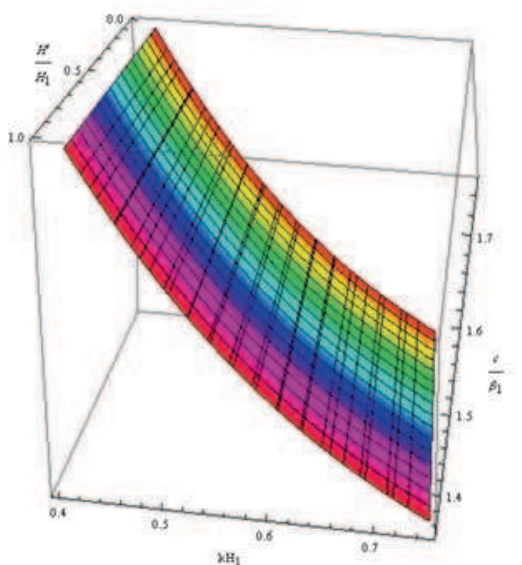

(i)

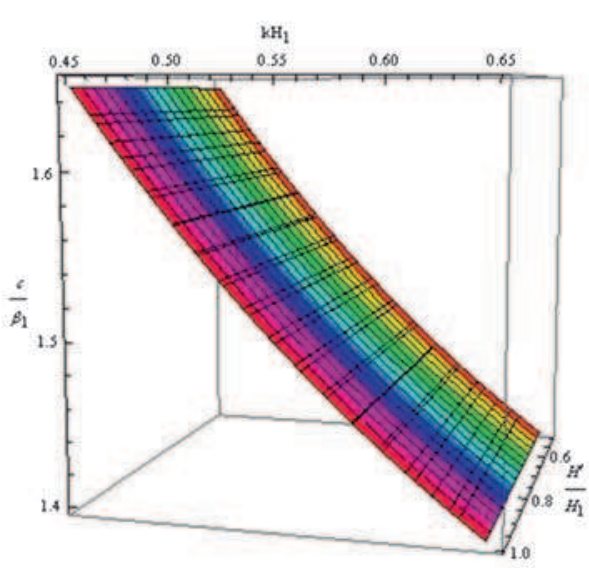

(ii)

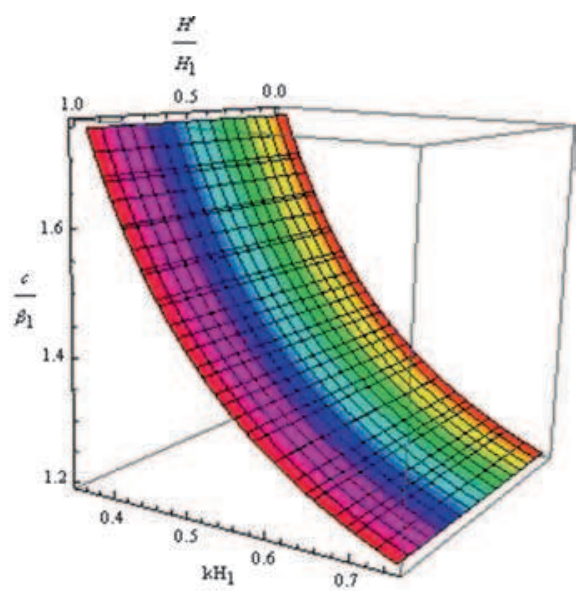

(iii)

Figure 12. Phase velocity $\left(c / \beta_{1}\right)$ against wave number $\left(k H_{1}\right)$ and irregularity parameter $\left(H^{\prime} / H_{1}\right)$ when parabolic irregularity is considered and $H_{2} / H_{1}=1.5, v H_{1}=0.1$; for (i) $\xi_{1}=0.1, \xi_{2}=0.1, \xi_{3}=0.1$, (ii) $\xi_{1}=0, \xi_{2}=0, \xi_{3}=0$; (iii) $\xi_{1}=-0.1, \xi_{2}=-0.1, \xi_{3}=-0.1$. 
respectively present at the interface of uppermost layer and intermediate layer. More specifically, figures 9(i), 10(i), 11(i) and 12(i) correspond to the cases when all the media namely uppermost layer, intermediate layer and lowermost half-space, are acted upon by horizontal compressive initial stresses; figures 9(ii), 10(ii), 11(ii) and 12(ii) correspond to the cases when all the media are without initial stresses; and figures 9(iii), 10(iii), 11(iii) and 12(iii) concur to the cases when all the media are acted upon by horizontal tensile initial stresses.

Surface plots in figure 9(i, ii and iii), concerned with the case of rectangular irregularity, exhibit that as heterogeneity prevails in the uppermost layer, phase velocity of Love-type wave increases irrespective of the situation that medium is prestressed or not. Further, the presence of compressive initial stress in each of the medium supports it in addition. Surface plots in figure 10(i, ii and iii), associated with the case of parabolic irregularity, displays the same trend with respect to heterogeneity parameter and initial stress as in figure 9 . But comparative study of figures $9(\mathrm{i}-\mathrm{iii})$ and $10(\mathrm{i}-\mathrm{iii})$ establish that parabolic irregularity support more to the phase velocity as compared to rectangular irregularity.

Surface plots in figure 11(i, ii and iii) irradiate the case of rectangular irregularity whereas surface plots in figures 12 (i, ii and iii) correspond to the case of parabolic irregularity. It is evident from these figures that initial stress and size of irregularity have a substantial effect on the phase velocity of Love-type wave. These surface plots manifest that the growth in the size of irregularity at the common interface of double layer disfavour the phase velocity of Love-type wave irrespective of presence and absence of initial stress in the medium.

\section{Conclusions}

The current study articulates the propagation of Love-type wave in an initially stressed irregular vertically heterogeneous layer overlying an initially stressed isotropic layer and an initially stressed isotropic half-space. The heterogeneity in the upper layer is caused due to exponential variation in rigidity, density and initial stress in terms of space variables pointing vertically downward. It is established through the study that wave number, width ratio of the layers, horizontal compressive/ tensile initial stresses, heterogeneity parameter of the uppermost layer and the irregularity parameter associated with rectangular form of irregularity and parabolic form of irregularity have a considerable effect on the phase velocity. The outcomes of the present study, taking the irregularity in rectangular as well as parabolic form, can be encapsulated as follows:

- Wave number has significant effect on the phase velocity. It is observed that phase velocity decreases with increase in wave number.

- The heterogeneity parameter of the uppermost layer has a favourable effect on the phase velocity of Love-type wave.

- The horizontal compressive initial stresses have a favourable effect on phase velocity whereas the horizontal tensile initial stresses have an unfavourable effect on phase velocity of Lovetype wave.

- The width ratio of the layers and the irregularity parameter has an adverse effect on phase velocity of Love-type wave. More precisely, it can be quoted that phase velocity of Love-type wave decreases with increase in the size of irregularity exist in the structure and the width of the uppermost layer as compared to the intermediate layer in the structure.

- As a special case of the problem, obtained dispersion relation is matched with classical Lovewave equation. This leads to acquiescence of the validity of the problem.

- Underneath condition holds good in our study of propagation of Love-type wave in the said geometry:

$$
\beta_{1}<\beta_{2}<c<\beta_{3} \text { or } \beta_{2}<\beta_{1}<c<\beta_{3} .
$$

The above condition indicates that phase velocity of Love-type wave must be less than the shear wave velocity in lowermost half-space and greater than the shear wave velocity in both the layers.

The present study has their possible applications in the sphere of seismology, engineering geology, earthquake engineering and geophysics. Specifically in the field of seismology, the problems affiliated to waves and vibrations propagating through a medium under initial stress (inducing mechanical properties to the medium depending on the magnitude of the stress) and divergent irregularities are of great significance to seismologists and geophysicists to understand and predict the seismic behaviour at different geological situations like mountain basins, mountain roots, continental margins, etc.

\section{Acknowledgements}

The authors convey their sincere thanks to Indian School of Mines, Dhanbad for providing all the necessary facilities for research. The authors also gratefully acknowledge the reviewers for their comprehensive and constructive suggestions for the improvement of quality of the manuscript. 


\section{Appendix I}

$$
\begin{aligned}
& A_{0}=-B_{0} \frac{\left(2 T+v \tan T H_{2}\right)}{\left(2 T \tan T H_{2}-v\right)} \\
& A_{1}=-B_{1} \frac{\left(2 T+v \tan T H_{2}\right)}{\left(2 T \tan T H_{2}-v\right)} \\
& B_{0}=\frac{2 p_{2} \mu_{2} \mu_{3} e^{-p_{3} d}\left(2 T \tan T H_{2}-v\right)}{U(k)}, \\
& B_{1}=\frac{R_{1} \mu_{1}^{0}\left(-v \cos T H_{1}+2 T \sin T H_{1}\right) e^{-\frac{v}{2} H_{1}}+2 R_{2} \cos T H_{1} e^{\frac{v}{2} H_{1}}}{-2 \mu_{1}^{0} T}, \\
& C_{0}=\frac{\left(2 T \cos T H_{1}+v \tan T H_{2} \cos T H_{1}+2 T \tan T H_{2} \sin T H_{1}-v \sin T H_{1}\right)}{\left(\mu_{2} p_{2} \cos p_{2} H_{1}+\mu_{3} p_{3} \sin p_{2} H_{1}\right) U(k)} \\
& \times\left(-2 p_{2}^{2} \mu_{2}^{2} \mu_{3} e^{-p_{3} d} e^{\frac{v}{2} H_{1}}\right)+\frac{4 \mu_{3} \sin p_{2} H_{1} e^{-p_{3} d}}{\left(\mu_{2} p_{2} \cos p_{2} H_{1}+\mu_{3} p_{3} \sin p_{2} H_{1}\right)}, \\
& D_{0}=\frac{\left(2 T \cos T H_{1}+v \tan T H_{2} \cos T H_{1}+2 T \tan T H_{2} \sin T H_{1}-v \sin T H_{1}\right)}{\left(\mu_{2} p_{2} \cos p_{2} H_{1}+\mu_{3} p_{3} \sin p_{2} H_{1}\right) U(k)} \\
& \times 2 \mu_{2} p_{2} \mu_{3}^{2} e^{-p_{3} d} e^{\frac{v}{2} H_{1}}+\frac{4 \mu_{3} \cos p_{2} H_{1} e^{-p_{3} d}}{\left(\mu_{2} p_{2} \cos p_{2} H_{1}+\mu_{3} p_{3} \sin p_{2} H_{1}\right)}, \\
& E=\frac{\left(2 T \cos T H_{1}+v \tan T H_{2} \cos T H_{1}+2 T \tan T H_{2} \sin T H_{1}-v \sin T H_{1}\right)}{\left(\mu_{2} p_{2} \cos p_{2} H_{1}+\mu_{3} p_{3} \sin p_{2} H_{1}\right) U(k)} \\
& \times\left(-2 \mu_{2}^{2} p_{2}^{2} \mu_{3} e^{-p_{3} d} e^{\frac{v}{2} H_{1}}\right)-\frac{2\left(\mu_{2} p_{2} \cos p_{2} H_{1}-\mu_{3} p_{3} \sin p_{2} H_{1}\right) e^{-p_{3} d}}{\left(\mu_{2} p_{2} \cos p_{2} H_{1}+\mu_{3} p_{3} \sin p_{2} H_{1}\right) p_{3}} . \\
& Q_{1}=\frac{e^{\frac{v}{2} H_{1}}}{2}\left(2 \mu_{2} p_{2} T \cos T H_{1}\left(p_{2} \mu_{2} \sin T H_{1}-\mu_{3} p_{3} \cos p_{2} H_{1}\right)\right. \\
& \left.-v \mu_{2} p_{2} \sin T H_{1}\left(p_{2} \mu_{2} \sin p_{2} H_{1}-\mu_{3} p_{3} \cos p_{2} H_{1}\right)\right) \\
& -\left(\left(T^{2}+\frac{v^{2}}{4}\right) \sin T H_{1}\left(\mu_{2} p_{2} \cos p_{2} H_{1}+\sin p_{2} H_{1} \mu_{3} p_{3}\right)\right) e^{-\frac{v}{2} H_{1}} \mu_{1}^{0} \\
& Q_{2}=e^{\frac{v}{2} H_{1}}\left(\mu_{2} p_{2}\left(\mu_{2} p_{2} \sin p_{2} H_{1}-\mu_{3} p_{3} \cos p_{2} H_{1}\right)\left(\frac{v}{2} \cos T H_{1}+T \sin T H_{1}\right)\right) \\
& +\left(\cos T H_{1}\left(\mu_{2} p_{2} \cos p_{2} H_{1}+\mu_{3} p_{3} \sin p_{2} H_{1}\right)\left(\frac{v^{2}}{4}+T^{2} \cos T H_{1}\right)\right) e^{-\frac{v}{2} H_{1}} \mu_{1}^{0}
\end{aligned}
$$

$S_{1}=4 Q_{2} \mu_{1}^{0} \mu_{3} \mu_{2} p_{2} T^{2}\left(\mu_{2} p_{2} \cos p_{2} h_{1}+\mu_{3} p_{3} \sin p_{2} h_{1}\right)-Q_{2}\left(Q_{4}+Q_{6}+Q_{8}\right) H^{\prime}$,

$S_{2}=2 \mu_{1}^{0} \mu_{3} p_{2} \mu_{2} T\left(\mu_{2} p_{2} \cos p_{2} H_{1}+\mu_{3} p_{3} \sin p_{2} H_{1}\right)\left(2 T Q_{1}-Q_{2} v\right)-Q_{1}\left(Q_{4}+Q_{6}+Q_{8}\right) H^{\prime}-Q_{2}\left(Q_{3}+Q_{5}+Q_{7}\right) H^{\prime}$,

$S_{3}=2 Q_{1} v \mu_{1}^{0} \mu_{3} \mu_{2} p_{2} T\left(\mu_{2} p_{2} \cos p_{2} H_{1}+\mu_{3} p_{3} \sin p_{2} H_{1}\right)-Q_{1}\left(Q_{3}+Q_{5}+Q_{7}\right) H^{\prime}$.

$F_{1}=4 Q_{2} \mu_{1}^{0} \mu_{3} \mu_{2} p_{2} T^{2}\left(\mu_{2} p_{2} \cos p_{2} h_{1}+\mu_{3} p_{3} \sin p_{2} h_{1}\right)-Q_{2}\left(Q_{4}+Q_{6}+Q_{8}\right) H^{\prime \prime}$,

$F_{2}=2 \mu_{1}^{0} \mu_{3} p_{2} \mu_{2} T\left(2 Q_{1} T-v Q_{2}\right)\left(p_{2} \mu_{2} \cos p_{2} H_{1}+\mu_{3} p_{3} \sin p_{2} H_{1}\right)-Q_{1}\left(Q_{4}+Q_{6}+Q_{8}\right) H^{\prime \prime}-Q_{2}\left(Q_{3}+Q_{5}+Q_{7}\right) H^{\prime \prime}$,

$F_{3}=2 Q_{1} v \mu_{1}^{0} \mu_{3} \mu_{2} p_{2} T\left(\mu_{2} p_{2} \cos p_{2} H_{1}+\mu_{3} p_{3} \sin p_{2} H_{1}\right)-Q_{1}\left(Q_{3}+Q_{5}+Q_{7}\right) H^{\prime \prime}$. 


\section{Appendix II}

$Q_{3}=4 \mu_{2} p_{2} T^{2} \mu_{3} \mu_{1}^{0}\left(T\left(\mu_{2} p_{2} \cos p_{2} H_{1}+\mu_{3} p_{3} \sin p_{2} H_{1}\right)\right.$ $-v \cos T H_{1} \sin T H_{1}\left(\mu_{2} p_{2} \cos p_{2} H_{1}\right.$

$\left.\left.-\mu_{3} p_{3} \sin p_{2} H_{1}\right)\right)$,

$Q_{4}=v \mu_{2} p_{2} \mu_{3} \mu_{1}^{0}\left\{\left(\mu_{2} p_{2} \cos p_{2} H_{1}+\mu_{3} p_{3} \sin p_{2} H_{1}\right)\right.$ $\times\left(2 T^{2}+\cos ^{2} T H_{1}\left(v^{2}+4 T^{2}\right)\right)+2 v T \cos T H_{1}$

$\left.\times \sin T H_{1}\left(\mu_{3} p_{3} \sin p_{2} H_{1}-\mu_{2} p_{2} \cos p_{2} H_{1}\right)\right\}$, $Q_{5}=2 e^{-\frac{v}{2} H_{1}} \mu_{1}^{0} \mu_{3} p_{2}\left(-v \cos T H_{1}+2 T \sin T H_{1}\right) Q_{1}$

$+\mu_{1}^{0}\left(2 v \mu_{2} p_{2}^{2} T \mu_{3}\left(\mu_{2} p_{2} \sin p_{2} H_{1}+\mu_{3} p_{3} \cos p_{2} H_{1}\right)\right.$

$-\mu_{2} p_{2}^{2} \mu_{3} \cos T H_{1} \sin T H_{1}\left(\mu_{2} p_{2} \sin p_{2} H_{1}\right.$

$\left.\left.-\mu_{3} p_{3} \cos p_{2} H_{1}\right)\left(4 T^{2}-v^{2}\right)\right)$,

$Q_{6}=\mu_{2} p_{2}^{2} \mu_{3}\left(\mu_{2} p_{2} \sin p_{2} H_{1}-\mu_{3} p_{3} \cos p_{2} H_{1}\right)$

$\times\left(v^{2} \cos ^{2} T H_{1}-4 T^{2} \sin ^{2} T H_{1}\right)$

$-2 v \mu_{2} p_{2}^{2} T \mu_{3}^{2} p_{3} \cos p_{2} H_{1} \cos T H_{1} \sin T H_{1}$

$-2 Q_{2} \mu_{3} p_{2}\left(v \cos T H_{1}+2 T \sin T H_{1}\right)$,

$Q_{7}=-4 e^{v H_{1}} \mu_{2}^{2} p_{2}^{3} T \mu_{3} \cos ^{2} T H_{1}$

$\times\left(\mu_{2} p_{2} \cos p_{2} H_{1}+\mu_{3} p_{3} \sin p_{2} H_{1}\right)$,

$Q_{8}=-2 e^{v H_{1}} \mu_{2} p_{2}^{3} \mu_{3} \cos T H_{1}$

$\times\left(\mu_{2} p_{2} \cos p_{2} H_{1}+\mu_{3} p_{3} \sin p_{2} H_{1}\right)$

$\times\left(v \mu_{2} \cos T H_{1}+2 \sin T H_{1}\right)$

$S_{4}=4 L_{2} \mu_{1}^{0} \mu_{3} \mu_{2} P_{2} t^{2}\left(1+\tan ^{2} t k H_{1}\right)$

$\times\left(\mu_{2} P_{2}+\mu_{3} P_{3} \tan P_{2} k H_{1}\right)-L_{2}\left(L_{4}+L_{6}+L_{8}\right) H^{\prime}$,

$S_{5}=2 \mu_{1}^{0} \mu_{3} P_{2} \mu_{2} t\left(1+\tan ^{2} t k H_{1}\right)$

$\times\left(P_{2} \mu_{2}+\mu_{3} P_{3} \tan P_{2} k H_{1}\right)$

$\times\left(2 L_{1} t-L_{2} \frac{v}{k}\right)-L_{1}\left(L_{4}+L_{6}+L_{8}\right) H^{\prime}$,

$S_{6}=2 L_{1} \frac{v}{k} \mu_{1}^{0} \mu_{3} \mu_{2} P_{2} t\left(1+\tan ^{2} t k H_{1}\right)$

$\times\left(\mu_{2} P_{2}+\mu_{3} P_{3} \tan P_{2} k H_{1}\right)$

$-L_{1}\left(L_{3}+L_{5}+L_{7}\right) H^{\prime}$,

$F_{4}=4 L_{2} \mu_{1}^{0} \mu_{3} \mu_{2} P_{2} t^{2}\left(1+\tan ^{2} t k H_{1}\right)$

$\times\left(\mu_{2} P_{2}+\mu_{3} P_{3} \tan P_{2} k H_{1}\right)$

$-L_{2}\left(L_{4}+L_{6}+L_{8}\right) H^{\prime \prime}$,

$F_{5}=2 \mu_{1}^{0} \mu_{3} \mu_{2} P_{2} t\left(1+\tan ^{2} t k H_{1}\right)$

$\times\left(\mu_{2} P_{2}+P_{3} \mu_{3} \tan P_{2} k H_{1}\right)\left(2 L_{1} t-L_{2} \frac{v}{k}\right)$

$-L_{1}\left(L_{4}+L_{6}+L_{8}\right) H^{\prime \prime}$,

$F_{6}=2 L_{1} \frac{v}{k} \mu_{1}^{0} \mu_{3} \mu_{2} P_{2} t\left(1+\tan ^{2} t k H_{1}\right)$

$\times\left(\mu_{2} P_{2}+\mu_{3} P_{3} \tan P_{2} k H_{1}\right)$

$-L_{1}\left(L_{3}+L_{5}+L_{7}\right) H^{\prime \prime}$,

$$
L_{1}=e^{\frac{v}{2} H_{1}} \mu_{2} P_{2}\left(P_{2} \mu_{2} \tan P_{2} k H_{1}+\mu_{3} P_{3}\right)
$$$$
\times\left(t-\frac{v}{2 k} \tan t k H_{1}\right)-e^{-\frac{v}{2} H_{1}} \mu_{1}^{0} \tan t k H_{1}
$$$$
\times\left(\mu_{2} P_{2}+\mu_{3} P_{3} \tan P_{2} k H_{1}\right)\left(t^{2}+\frac{v^{2}}{4 k^{2}}\right),
$$

$L_{2}=e^{\frac{v}{2} H_{1}} \mu_{2} P_{2}\left(\mu_{2} P_{2} \tan P_{2} k H_{1}-\mu_{3} P_{3}\right)$

$\times\left(\frac{v}{2 k}+t \tan t k H_{1}\right)+e^{-\frac{v}{2} H_{1}} \mu_{1}^{0}$

$\times\left(\mu_{2} P_{2}+\mu_{3} P_{3} \tan P_{2} k H_{1}\right)\left(\frac{v^{2}}{4 k^{2}}+t^{2}\right)$

$$
L_{3}=4 \mu_{2} P_{2} t^{3} \mu_{3} \mu_{1}^{0}\left(1+\tan ^{2} t k H_{1}\right)
$$

$\times\left(\mu_{2} P_{2}+\mu_{3} P_{3} \tan P_{2} k H_{1}\right)-4 \frac{v}{k} \mu_{2} P_{2} t^{2} \mu_{3} \mu_{1}^{0}$

$\times \tan t k H_{1}\left(\mu_{2} P_{2}-\mu_{3} P_{3} \tan P_{2} k H_{1}\right)$,

$L_{4}=\frac{v}{k} \mu_{2} P_{2} \mu_{3} \mu_{1}^{0}\left(\mu_{3} P_{3} \tan P_{2} k H_{1}+\mu_{2} P_{2}\right)$

$\times\left(2 t^{2}\left(1+\tan ^{2} t k H_{1}\right)+\frac{v^{2}}{k^{2}}+4 t^{2}\right)$

$+2 \frac{v^{2}}{k^{2}} \mu_{2} P_{2} t \mu_{3} \mu_{1}^{0} \tan t k H_{1}\left(\mu_{3} P_{3} \tan P_{2} k H_{1}-\mu_{2} P_{2}\right)$,

$L_{5}=2 e^{-\frac{v}{2} H_{1}} \mu_{1}^{0} \mu_{3} P_{2}\left(-\frac{v}{k}+2 t \tan t k H_{1}\right) L_{1}+\mu_{1}^{0}$

$\times\left(2 \frac{v}{k} \mu_{2} P_{2}^{2} t \mu_{3} \tan ^{2} t k H_{1}\left(\mu_{3} P_{3}+\mu_{2} P_{2} \tan P_{2} k H_{1}\right)\right.$

$+\mu_{2} P_{2}^{2} \mu_{3}\left(2 \frac{v}{k} t-\tan t k H_{1}\left(\frac{v^{2}}{k^{2}}+4 t^{2}\right)\right)$

$\left.\times\left(\mu_{2} P_{2} \tan P_{2} k H_{1}-\mu_{3} P_{3}\right)\right)$,

$L_{6}=\left(\frac{v^{2}}{k^{2}} \mu_{2} P_{2}^{2} \mu_{3}-4 \mu_{2} P_{2}^{2} t^{2} \mu_{3} \tan ^{2} t k H_{1}\right)$

$\times\left(\mu_{2} P_{2} \tan P_{2} k H_{1}-\mu_{3} P_{3}\right)$

$-2 \mu_{3} P_{2}\left(\frac{v}{k} \mu_{2} P_{2} t \mu_{3} P_{3} \tan t k H_{1}\right.$

$\left.+L_{2}\left(\frac{v}{k}-4 t \tan t k H_{1}\right)\right)$,

$L_{7}=-4 e^{v H_{1}} \mu_{2}^{2} P_{2}^{3} t \mu_{3}\left(\mu_{2} P_{2}+\mu_{3} P_{3} \tan P_{2} k H_{1}\right)$,

$L_{8}=-2 e^{v H_{1}} \mu_{2} P_{2}^{3} \mu_{3}\left(\mu_{2} P_{2}+P_{3} \mu_{3} \tan P_{2} k H_{1}\right)$

$\times\left(\frac{v}{k} \mu_{2}+2 \tan t k H_{1}\right)$,

$S_{4}^{(1)}=4 L_{2}^{(1)} \mu_{1}^{0} \mu_{3} \mu_{2} P_{2}\left(t^{(1)}\right)^{2}\left(1+\tan ^{2} t^{(1)} k H_{1}\right)$

$\times\left(\mu_{2} P_{2}+\mu_{3} P_{3} \tan P_{2} k H_{1}\right)$

$-L_{2}^{(1)}\left(L_{6}^{(1)}+L_{8}^{(1)}\right) H^{\prime}$,

$S_{5}^{(1)}=4 L_{1}^{\prime} \mu_{1}^{0} \mu_{3} P_{2} \mu_{2}\left(t^{(1)}\right)^{2}\left(1+\tan ^{2} t^{(1)} k H_{1}\right)$

$\times\left(P_{2} \mu_{2}+\mu_{3} P_{3} \tan P_{2} k H_{1}\right)$

$-L_{1}^{(1)}\left(L_{6}^{(1)}+L_{8}^{(1)}\right) H^{\prime}$,

$S_{6}^{(1)}=-L_{1}^{(1)}\left(L_{3}^{(1)}+L_{5}^{(1)}+L_{7}^{(1)}\right) H^{\prime}$, 


$$
\begin{aligned}
& F_{4}^{(1)}=4 \mu_{1}^{0} \mu_{3} L_{2}^{(1)} \mu_{2} P_{2}\left(t^{(1)}\right)^{2}\left(1+\tan ^{2} t^{(1)} k H_{1}\right) \\
& \times\left(\mu_{2} P_{2}+\mu_{3} P_{3} \tan P_{2} k H_{1}\right) \\
& -L_{2}^{(1)}\left(L_{6}^{(1)}+L_{8}^{(1)}\right) H^{\prime \prime}, \\
& F_{5}^{(1)}=4 \mu_{1}^{0} \mu_{3} L_{1}^{(1)} P_{2} \mu_{2}\left(t^{(1)}\right)^{2}\left(1+\tan ^{2} t^{(1)} k H_{1}\right) \\
& \times\left(P_{2} \mu_{2}+\mu_{3} P_{3} \tan P_{2} k H_{1}\right) \\
& -L_{1}^{(1)}\left(L_{6}^{(1)}+L_{8}^{(1)}\right) H^{\prime \prime}, \\
& F_{6}^{(1)}=-L_{1}^{(1)}\left(L_{3}^{(1)}+L_{5}^{(1)}+L_{7}^{(1)}\right) H^{\prime \prime}, \\
& L_{1}^{(1)}=\mu_{2} P_{2} t^{(1)}\left(P_{2} \mu_{2} \tan P_{2} k H_{1}+\mu_{3} P_{3}\right)-\mu_{1}^{0}\left(t^{(1)}\right)^{2} \\
& \times \tan t^{(1)} k H_{1}\left(\mu_{2} P_{2}+\mu_{3} P_{3} \tan P_{2} k H_{1}\right), \\
& L_{2}^{(1)}=\mu_{2} P_{2} t^{(1)} \tan t^{(1)} k H_{1}\left(\mu_{2} P_{2} \tan P_{2} k H_{1}-\mu_{3} P_{3}\right) \\
& +\mu_{1}^{0}\left(t^{(1)}\right)^{2}\left(\mu_{2} P_{2}+\mu_{3} P_{3} \tan P_{2} k H_{1}\right) \\
& L_{3}^{(1)}=4 \mu_{2} P_{2} \mu_{3} \mu_{1}^{0}\left(t^{(1)}\right)^{3}\left(1+\tan ^{2} t^{(1)} k H_{1}\right) \\
& \times\left(\mu_{2} P_{2}+\mu_{3} P_{3} \tan P_{2} k H_{1}\right), \\
& L_{5}^{(1)}=4 \mu_{1}^{0} \mu_{3} P_{2} t^{(1)} \tan t^{(1)} k H_{1} \\
& \times\left(L_{1}-\mu_{2} P_{2} t^{(1)}\left(\mu_{2} P_{2} \tan P_{2} k H_{1}-\mu_{3} P_{3}\right)\right), \\
& L_{6}^{(1)}=4 \mu_{3} P_{2} t^{(1)} \tan t^{(1)} k H_{1}\left(L_{2}^{(1)}-\mu_{2} P_{2} t^{(1)}\right. \\
& \left.\times \tan t^{(1)} k H_{1}\left(\mu_{2} P_{2} \tan P_{2} k H_{1}-\mu_{3} P_{3}\right)\right), \\
& L_{7}^{(1)}=-4 \mu_{2}^{2} P_{2}^{3} t^{(1)} \mu_{3}\left(\mu_{2} P_{2}+\mu_{3} P_{3} \tan P_{2} k H_{1}\right), \\
& L_{8}^{(1)}=-4 \mu_{2} P_{2}^{3} \mu_{3} \tan t^{(1)} k H_{1} \\
& \times\left(\mu_{2} P_{2}+P_{3} \mu_{3} \tan P_{2} k H_{1}\right), \\
& S_{4}^{(2)}=4 L_{2}^{(2)} \mu_{1}^{0} \mu_{3} \mu_{2} P_{2}^{(1)}\left(t^{(1)}\right)^{2}\left(1+\tan ^{2} t k H_{1}\right) \\
& \times\left(\mu_{2} P_{2}^{(1)}+\mu_{3} P_{3}^{(1)} \tan P_{2}^{(1)} k H_{1}\right) \\
& -L_{2}^{(2)}\left(L_{6}^{(2)}+L_{8}^{(2)}\right) H^{\prime}, \\
& S_{5}^{(2)}=4 L_{1}^{(2)} \mu_{1}^{0} \mu_{3} \mu_{2} P_{2}^{(1)}\left(t^{(1)}\right)^{2}\left(1+\tan ^{2} t^{(1)} k H_{1}\right) \\
& \times\left(P_{2}^{(1)} \mu_{2}+\mu_{3} P_{3}^{(1)} \tan P_{2}^{(1)} k H_{1}\right) \\
& -L_{1}^{(2)}\left(L_{6}^{(2)}+L_{8}^{(2)}\right) H^{\prime}, \\
& S_{6}^{(2)}=-L_{1}^{(2)}\left(L_{3}^{(2)}+L_{5}^{(2)}+L_{7}^{(2)}\right) H^{\prime}, \\
& F_{4}^{(2)}=4 L_{2}^{(2)} \mu_{1}^{0} \mu_{3} \mu_{2} P_{2}^{(1)}\left(t^{(1)}\right)^{2}\left(1+\tan ^{2} t^{(1)} k H_{1}\right) \\
& \times\left(\mu_{2} P_{2}^{(1)}+\mu_{3} P_{3}^{(1)} \tan P_{2}^{(1)} k H_{1}\right) \\
& -L_{2}^{(2)}\left(L_{6}^{(2)}+L_{8}^{(2)}\right) H^{\prime \prime}, \\
& F_{5}^{(2)}=4 L_{1}^{(2)} \mu_{1}^{0} \mu_{3} P_{2}^{(1)} \mu_{2}\left(t^{(1)}\right)^{2}\left(1+\tan ^{2} t^{(1)} k H_{1}\right) \\
& \times\left(P_{2}^{(1)} \mu_{2}+\mu_{3} P_{3}^{(1)} \tan P_{2}^{(1)} k H_{1}\right) \\
& -L_{1}^{(2)}\left(L_{6}^{(2)}+L_{8}^{(2)}\right) H^{\prime \prime}, \\
& F_{6}^{(2)}=-L_{1}^{(2)}\left(L_{3}^{(2)}+L_{5}^{(2)}+L_{7}^{(2)}\right) H^{\prime \prime}, \\
& L_{1}^{(2)}=\mu_{2} P_{2}^{(1)} t^{(1)}\left(\mu_{2} P_{2}^{(1)} \tan P_{2}^{(1)} k H_{1}-\mu_{3} P_{3}^{(1)}\right) \\
& -\mu_{1}^{0}\left(t^{(1)}\right)^{2} \tan t^{(1)} k H_{1} \\
& \times\left(\mu_{2} P_{2}^{(1)}-\mu_{3} P_{3}^{(1)} \tan P_{2}^{(1)} k H_{1}\right),
\end{aligned}
$$

$$
\begin{aligned}
L_{2}^{(2)}= & \mu_{2} P_{2}^{(1)} t^{(1)} \tan t^{(1)} k H_{1}\left(\mu_{2} P_{2}^{(1)} \tan P_{2}^{(1)} k H_{1}\right. \\
& \left.-\mu_{3} P_{3}^{(1)}\right)+\mu_{1}^{0}\left(t^{(1)}\right)^{2}\left(\mu_{2} P_{2}^{(1)}\right. \\
& \left.+\mu_{3} P_{3}(1) \tan P_{2}^{(1)} k H_{1}\right) \\
L_{3}^{(2)}= & 4 \mu_{2} P_{2}^{(1)}\left(t^{(1)}\right)^{3} \mu_{3} \mu_{1}^{0}\left(1+\tan ^{2} t^{(1)} k H_{1}\right) \\
& \times\left(\mu_{2} P_{2}^{(1)}+\mu_{3} P_{3}^{(1)} \tan P_{2}^{(1)} k H_{1}\right), \\
L_{5}^{(2)}= & 4 \mu_{1}^{0} \mu_{3} P_{2}^{(1)} t^{(1)} \tan t^{(1)} k H_{1}\left(L_{1}-P_{2}^{(1)} \mu_{2} t^{(1)}\right. \\
& \left.\times\left(\mu_{2} P_{2}^{(1)} \tan P_{2}^{(1)} k H_{1}-\mu_{3} P_{3}^{(1)}\right)\right), \\
L_{6}^{(2)}= & 4 \mu_{3} P_{2}^{(1)} t^{(1)} \tan t^{(1)} k H_{1}\left(L_{2}^{(2)}-\mu_{2} P_{2}^{(1)} t^{(1)}\right. \\
& \left.\times \tan t^{(1)} k H_{1}\left(\mu_{2} P_{2}^{(1)} \tan P_{2}^{(1)} k H_{1}-\mu_{3} P_{3}^{(1)}\right)\right), \\
L_{7}^{(2)}= & -4 \mu_{2}^{2} \mu_{3}\left(P_{2}^{(1)}\right)^{3} t^{(1)} \\
& \times\left(\mu_{2} P_{2}^{(1)}+\mu_{3} P_{3}^{(1)} \tan P_{2}^{(1)} k H_{1}\right), \\
L_{8}^{(2)}= & -4 \mu_{2}\left(P_{2}^{(1)}\right)^{3} \mu_{3} \tan t^{(1)} k H_{1} \\
& \times\left(\mu_{2} P_{2}^{(1)}+P_{3}^{(1)} \mu_{3} \tan P_{2}^{(1)} k H_{1}\right), \\
P_{2}^{(1)}= & \sqrt{\frac{c^{2}}{\beta_{2}^{2}}-1, \quad P_{3}^{(1)}=\sqrt{1-\frac{c^{2}}{\beta_{3}^{2}}} .} \\
t^{(2)}= & \sqrt{\frac{c^{2}}{\beta_{2}^{2}}-1+\xi}, \quad t^{(1)}=\sqrt{\beta_{2}^{2}}, \\
&
\end{aligned}
$$

\section{References}

Acharya D P and Roy I 2009 Effect of surface stress and irregularity of the interface on the propagation of SHwaves in the magneto-elastic crustal layer based on a solid semi space; Sadhana 34(2) 309-330.

Acharya D P, Roy I and Sengupta S 2009 Effect of magnetic field and initial stress on the propagation of interface waves in transversely isotropic perfectly conducting media; Acta Mech. 202(1-4) 35-45.

Ben-Hador R and Buchen P 1999 Love and Rayleigh waves in non-uniform media; Geophys. J. Int. 137(2) 521534.

Bhattacharya J 1969 The possibility of the propagation of Love-type waves in an intermediate heterogeneous layer lying between two semi-infinite isotropic homogeneous elastic layers; Pure Appl. Geophys. 72(1) 61-71.

Biot M A 1965 Mechanics of Incremental Deformations; Wiley, New York.

Chattaraj R, Samal S K and Mahanti N C 2012 Dispersion of Love wave propagating in irregular anisotropic porous stratum under initial stress; Int. J. Geomech. 13(4) 402-408.

Chattopadhyay A and De R K 1983 Love type waves in a porous layer with irregular interface; Int. J. Eng. Sci. 21 1295-1303.

Chattopadhyay A and Pal A K 1983 Dispersion curves of SH-waves caused by irregularity in the pre-stressed internal stratum; Acta Geophys. 31(1) 37-49. 
Chattopadhyay A and Singh A K 2012 Propagation of magnetoelastic shear waves in an irregular self-reinforced layer; J. Eng. Math. 75(1) 139-155.

Chattopadhyay A, Chakraborty M and Pal A K 1983 Effects of irregularity on the propagation of guided SH-waves; J. Theor. Appl. Mech. 2(2) 215-225.

Chattopadhyay A, Gupta S, Sahu S A and Singh A K 2011a Dispersion equation of magnetoelastic shear waves in irregular monoclinic layer; J. Appl. Math. Mech. 32(5) $571-586$.

Chattopadhyay A, Gupta S, Sahu S A and Singh A K 2011b Dispersion equation of magnetoelastic shear waves in an irregular monoclinic layer; J. Appl. Math. Mech. 32(5) $571-586$.

Dey S and Addy S K 1978 Love waves under initial stresses; Acta Geophys. 24(1) 47.

Dutta S 1963 Love waves in a non-homogeneous internal stratum lying between two semi-infinite isotropic media; Pure Appl. Geophys. 28 156-160.
Ewing W M and Press W S F 1957 Elastic Waves in Layered Media; McGraw-Hill, New York, NY, USA.

Gubbins D 1990 Seismology and Plate Tectonics; Cambridge University Press, Cambridge.

Kaur T, Singh A K, Chattopadhyay A and Sharma S K 2014 Dynamic response of normal moving load on an irregular fiber-reinforced half-space; J. Vib. Control., doi: 1077546314528525.

Khurana P and Vashisth A K 2001 Love wave propagation in a pre-stressed medium; Indian J. Pure Appl. Math. 32(8) 1201-1207.

Sato Y 1952 Love waves propagated upon heterogeneous medium; Bull. Earthquake Res. Inst. 30 1-12.

Singh S S 2011 Love wave in a layer medium bounded by irregular boundary surfaces; J. Vib. Control. 17 789-795.

Tranter C J 1966 Integral Transform in Mathematical Physics; Methuen and Co. Ltd., London.

Willis H F 1948 A formula for expanding an integral as a series; Philos. Mag. 39(293) 455-459. 